\title{
An expanded stratigraphic record of the Devonian- Carboniferous boundary Hangenberg biogeochemical Event from Southeast lowa (U.S.A.)
}

\author{
Brittany M. Stolfus, Bradley D. Cramer, Ryan J. Clark, Nicholas J. Hogancamp, \\ James E. Day, Stephanie A. Tassier-Surine \& Brian J. Witzke
}

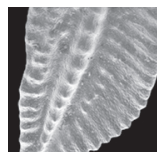

\begin{abstract}
The Devonian-Carboniferous boundary in the type area of the Mississippian subsystem (tri-state area of Iowa, Illinois, and Missouri) has been historically difficult to identify. Many of the localities contain similar lithologies and stratigraphic successions, but chronostratigraphic correlation of seemingly identical lithologies can vary greatly in this interval and frequently this has led to miscorrelation. In particular, the similar lithofacies that comprise the McCraney Formation and Louisiana Formation have been a source of stratigraphic confusion for over 100 years. To investigate the Devonian-Carboniferous boundary interval in the Mississippian type area we selected two localities in southeastern Iowa, the H-28 core from Lee County outside of Keokuk, Iowa, and the Starr's Cave outcrop located near Burlington, Iowa. In total, 62 conodont samples and 299 carbonate carbon isotope samples were processed for this study and recorded the Hangenberg positive carbon isotope excursion and 25 conodont species, including a diverse assemblage of siphonodellids. The Hangenberg excursion is recorded in over $20 \mathrm{~m}$ of strata in southeast Iowa, making this one of the thickest stratigraphic records of this important biogeochemical event yet recovered, and helps to define more clearly the position of the base of the Carboniferous System in the region. These results show that the "McCraney" Fm. at the Starr's Cave outcrop and the coeval carbonate unit in the H-28 core are both the Louisiana Formation, and calls into question the use of the name McCraney throughout the State of Iowa. $\bullet$ Key words: conodont, carbon isotope, Siphonodella, Louisiana Limestone, Devonian-Carboniferous boundary.
\end{abstract}

Stolfus, B.M., Cramer, B.D., Clark, R.J., Hogancamp, N.J., Day, J.E., Tassier-Surine, S.A. \& Witzke, B.J. 2020. An expanded stratigraphic record of the Devonian-Carboniferous boundary Hangenberg biogeochemical Event from Southeast Iowa (U.S.A.). Bulletin of Geosciences 95(4), 469-495 (18 figures, 2 tables). Czech Geological Survey, Prague. ISSN 1214-1119. Manuscript received January 15, 2020; accepted in revised form October 9, 2020; published online November 15, 2020; issued November 15, 2020.

Brittany M. Stolfus, Bradley D. Cramer \& Brian J. Witzke, Department of Earth \& Environmental Sciences, University of Iowa, Iowa City, Iowa, USA; brittany-stolfus@uiowa.edu・Ryan J. Clark \& Stephanie A. Tassier-Surine, Iowa Geological Survey, University of Iowa, Iowa City, Iowa, USA • Nicholas J. Hogancamp, Hess Corporation, Houston, Texas, USA \& Department of Earth \& Atmospheric Sciences, University of Houston, Houston, Texas, USA • James E. Day, Department of Geography, Geology, and the Environment, Illinois State University, Normal, Illinois, USA

The Devonian-Carboniferous boundary (DCB) interval coincides with a mass extinction on the scale of the 'Big Five' (Sepkoski 1996, Kaiser et al. 2016) and a major perturbation to the global carbon cycle (Cramer et al. 2008, Saltzman \& Thomas 2012). The DCB strata of the tri-state area of Missouri, Illinois, and Iowa have been studied for over a century and contain historically important strata for the type Mississippian area including the type area of the lowest Carboniferous North American Kinderhookian Stage (global lower Tournaisian Stage) of the Mississippian Subsystem. The majority of this work occurred more than 50 years ago (e.g., Scott \& Collinson 1961) with more recent work limited to the late 1990's (Chauffe \& Nichols 1995, Witzke \& Bunker 1996, Chauffe \&
Guzman 1997). However, significant problems with unit correlation remain due to long standing nomenclature divides across state boundaries, lack of study, or lowresolution sampling.

Two units of strikingly similar lithologies, the McCraney Formation and the Louisiana Formation, are critical to the placement of the DCB in the tri-state area. These units often occur within a few miles of one another; however, they have never been identified in the same succession, either in outcrop or in the subsurface. Historically, a nearly equal number of publications have considered these units to be equivalent (e.g., Weller 1900, Weller \& Sutton 1940, Harris 1947, Stainbrook 1950) as have considered them to be temporally distinct (Keyes 1895, Weller 1906, 


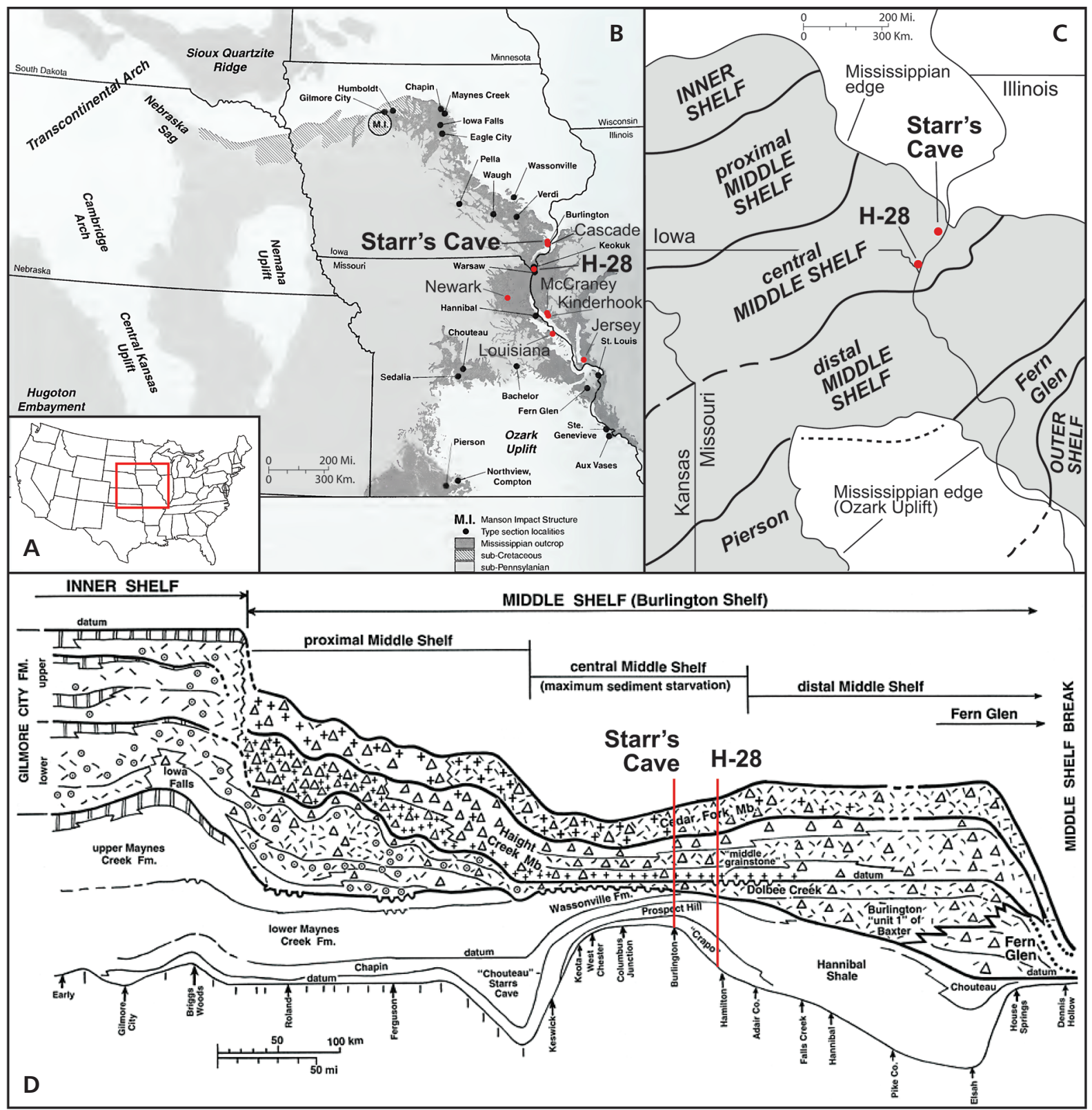

Figure 1. A, B - distribution of Mississippian outcrops in the tri-State area of Missouri, Illinois, and Iowa, including the two locations (H-28 and Starr's Cave) sampled in this study. Other localities shown and discussed in the text include Cascade Station (Cascade), Teneriffe School (Jersey), McCraney North (McCraney), the town of Kinderhook (all from Scott \& Collinson, 1961), Fabius River Bridge and Fabius River Cut (Newark) from Chauffe \& Guzman (1997), and the town of Louisiana (e.g., Cramer et al. 2008). Modified from Witzke \& Bunker (2001). • C - paleoceanographic reconstruction of the tri-state area during the Mississippian. $\bullet$ D - cross section of Kinderhookian strata spanning northwest to southeast from Early to Dennis Hollow. Sampling localities examined in this study are marked with a red line. B and C modified from Witzke \& Bunker (2002).

Moore 1928, Laudon 1931, Williams 1943, Thomas 1949, Workman \& Gillette 1956, Scott \& Collinson 1961). These different correlation interpretations started in Missouri and Illinois and then later spread into southeastern Iowa when Moore (1928) correlated the basal Mississippian carbonate unit exposed at Burlington, Iowa (IA), with the McCraney Formation. This practice has continued within the State of Iowa into the $21^{\text {st }}$ Century (Witzke et al. 1990, Witzke \& Bunker 2001).
In the wake of improved conodont biostratigraphic information from the Louisiana and the McCraney in the tri-state area (Chauffe \& Nichols 1995, Chauffe \& Guzman 1997), and following the preparation of multiple field trip guidebooks to the region (Heckel 2001, Witzke et al. 2002), the correlation of these beds in southeast Iowa with the McCraney Formation came into question. Witzke \& Bunker (2002) summarized these issues in southeastern Iowa and began using the term "McCraney" 
Formation. Here we present integrated high-resolution biochemostratigraphy of conodonts and carbon isotopes from southeastern Iowa to improve our understanding of this unit and its regional correlation. In total, 62 conodont samples and 299 carbon isotope samples were collected and processed that help to improve stratigraphic correlation in the type area of the Mississippian, identify the position of the Hangenberg crisis in Iowa, and demonstrate the likely position of the DCB in type Mississippian strata throughout the region.

\section{Geologic Setting}

During the Mississippian, a broad epicontinental sea covered the U.S. Mid-continent and the tri-state area of Iowa, Missouri, and Illinois was located between 10-20 south latitude (e.g., Lane 1978, Witzke \& Bunker 1996). The expansive Mississippian carbonate platform known as the Burlington Shelf, transitions to outer shelf and deeper water deposits towards southern Illinois and southeastern Missouri (Fig. 1). The samples included in this study are from southeastern Iowa within the central middle shelf and include an outcrop at Starr's Cave Park and Preserve north of Burlington, Iowa, and the H-28 core near Keokuk, Iowa. The central middle shelf and sections of study are within the area of maximum sediment starvation across the Burlington Shelf, however, the outcrops in southeastern Iowa are historically important for regional stratigraphy (e.g., Moore 1928, Scott \& Collinson 1961).

Mississippian strata increase in thickness from the central middle shelf towards the distal middle shelf (Fig. 2). The two localities studied were the Starr's Cave outcrop north of Burlington, IA and the H-28 core near Keokuk, IA. From Burlington to Keokuk, the lower Mississippian interval expands and doubles in thickness over less than 50 miles. However, the lower Mississippian interval expands even more as you travel southeast into Missouri and Illinois (Fig. 1C).

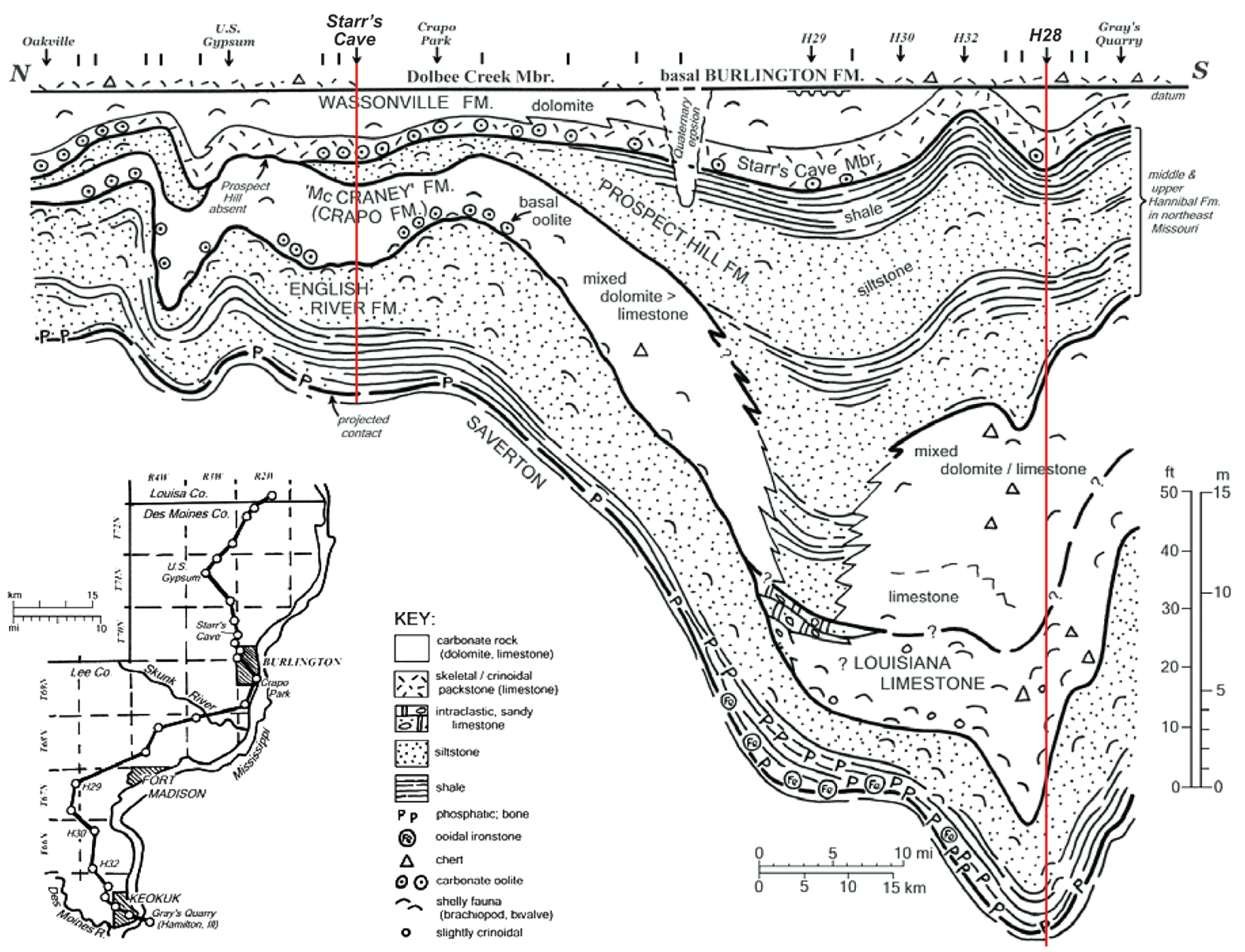

Figure 2. North-south cross section of Kinderhookian stratigraphy through southeastern Iowa illustrating the increase in stratigraphic thickness towards the south. The two locations sampled in this study are designated by red lines. Modified from Witzke \& Bunker (2002). 


\section{Devonian-Carboniferous boundary}

The DCB interval contains one of the largest mass extinctions in Earth history and impacted nearly all marine fauna including conodonts, ammonoids, trilobites, corals, sponges, brachiopods, ostracodes, marine phytoplankton, foraminifera, amphibians, and placoderms (Walliser 1984, 1996; Kaiser et al. 2011, 2016). This extinction event, known as the Hangenberg crisis, coincides with the onset one of the largest positive carbon isotope excursions of the Phanerozoic that reaches values greater than $+6.0 \%$ o (Cramer et al. 2008, Saltzman \& Thomas 2012). The stratigraphic record of the tri-state area of the U.S. Midcontinent has been under-evaluated during the past two decades with respect to the global importance of this biogeochemical event.

The Global Boundary Stratotype Section and Point (GSSP) of the base of the Carboniferous System is located at La Serre, Montagne Noire, France, and was chosen to coincide with the first appearance datum (FAD) of the conodont Siphonodella sulcata, which marks the base of the eponymous biozone (Paproth et al. 1991, Davydov et al. 2012). The base of the S. sulcata Zone occurs towards the end of the Hangenberg crisis, significantly above the onset of the Hangenberg positive carbon isotope excursion. The base of this zone has been notoriously difficult to correlate to global stratigraphy due in part to taxonomic uncertainty around the marker species (Kaiser \& Becker 2007, Kaiser \& Corradini 2008, Kaiser 2009, Davydov et al. 2012, Becker et al. 2016). As a result, this boundary is currently under review by a working group from the international subcommissions on Devonian and Carboniferous stratigraphy and there are several proposed biostratigraphic positions for the future base of the Carboniferous System (Becker et al. 2016, Corradini et al. 2017). All of the proposed revisions place the base of the Carboniferous lower, and the clearest conodontbased position is the base of the Protognathodus kockeli Zone (Becker et al. 2016, Spalletta et al. 2017, Corradini et al. 2017), although the full "Montpellier Criteria" for

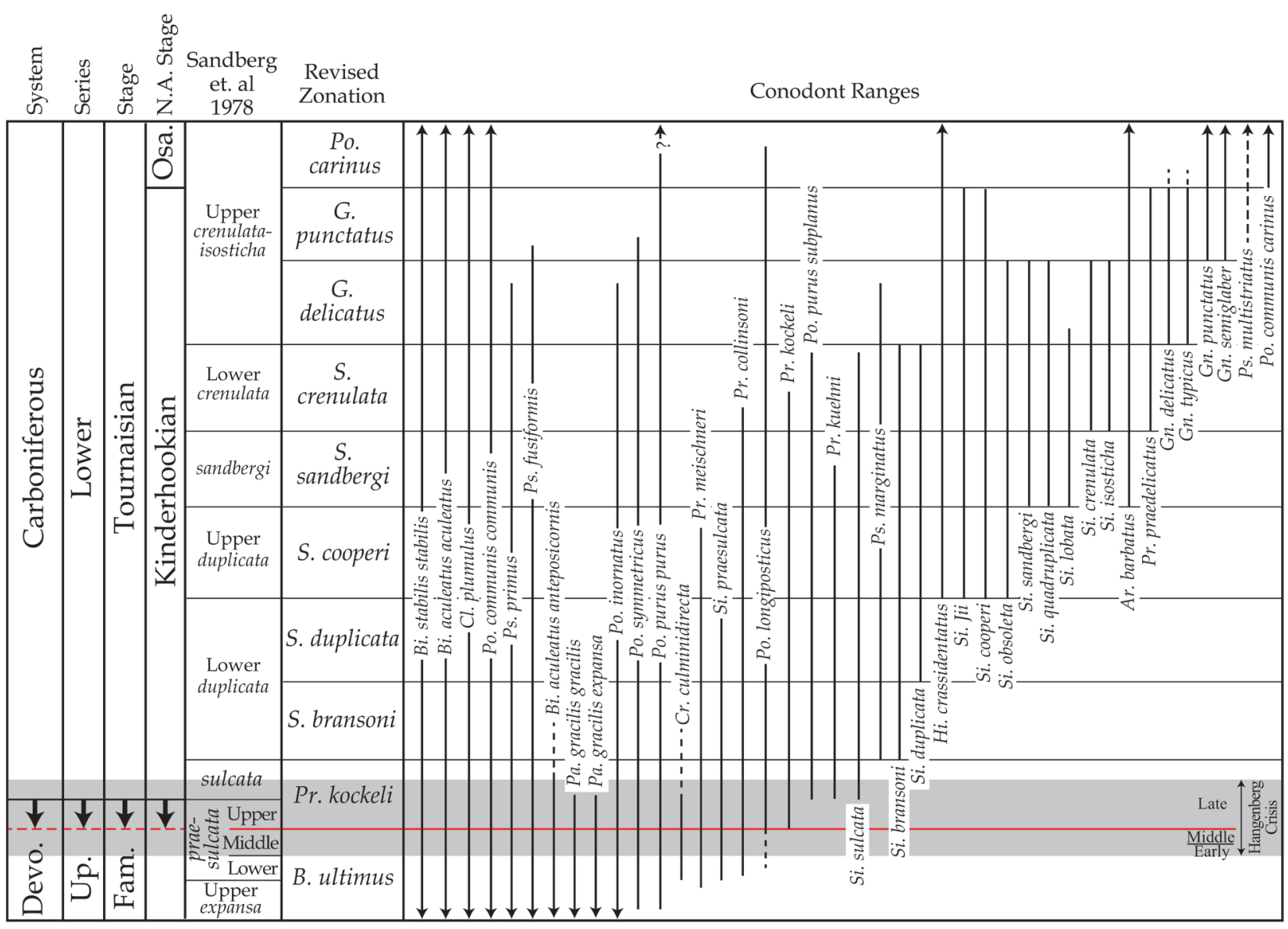

Figure 3. Revised conodont biozonation and range chart for the Kinderhookian. Traditional zonation of Sandberg et al. (1978) shown at left with comparison of new zones. New zonation is a composite of Kaiser et al. (2009), Spalletta et al. (2017), and Zhuravlev \& Plotitsyn (2017) and is after Hogancamp et al. (2019). The dashed line corresponds to the base of the Protognathodus kockeli Zone, indicating the likely position of the DevonianCarboniferous boundary according to the Montpellier Critera (see text). This also fits well with the proposed definition of the end of major regression. 

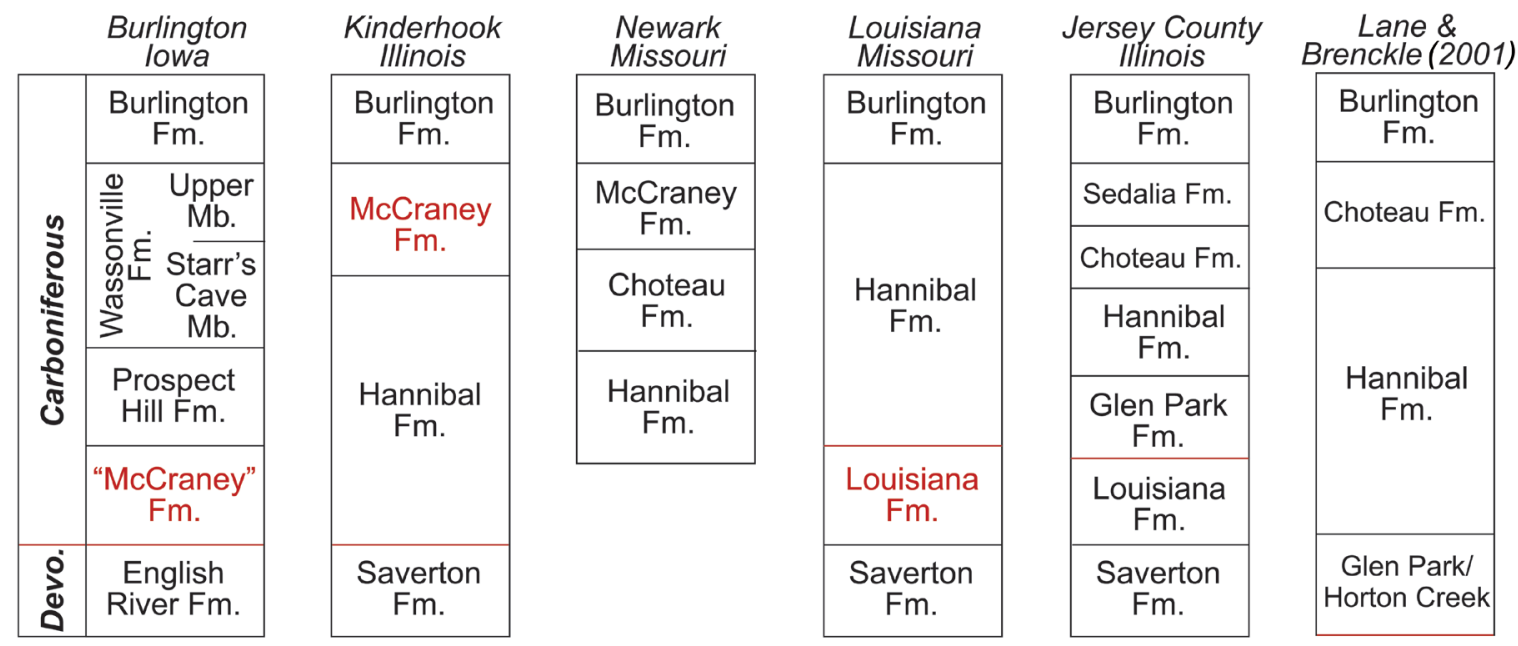

Figure 4. Comparison of Kinderhookian stratigraphic successions in the tri-state area, following Scott \& Collison (1961), Chauffe \& Guzman (1997), and Lane \& Brenckle (2001). Red lines designate traditional placements of the Devonian-Carboniferous boundary in each area.

a revised placement of the base Carboniferous GSSP include the base of the $P$. kockeli Zone, the beginning of post-extinction radiation, the top of major regression, and the end of mass extinction. Throughout this study the potential new placement (based herein upon the $P$. kockeli Zone and the top of major regression) is used for placement of the base Carboniferous System in our two studied sections (Fig. 3).

Here, we utilize a revised conodont biozonation that closely follows Kaiser et al. (2009) for the Kinderhookian, Zhuravlev \& Plotitsyn (2017) for the uppermost Kinderhookian-lowest Osagean, and Spalletta et al. (2017) for the DCB, with the following comments and changes. A complete discussion of the revisions to the Kinderhookian conodont biozonation utilized herein can be found in Hogancamp et al. (2019). The succession from $S$. bransoni to $S$. sandbergi is identical to Kaiser et al. (2009) with the exception of the Siphonodella cooperi Zone. As noted by Becker et al. (2016) the name Siphonodella hassi was rejected due to homonymy and replaced with Siphonodella jii. However, the designation of $S$. hassi by $\mathrm{Ji}$ (1985) was an invalid junior synonym of Siphonodella cooperi hassi (Thompson \& Fellows, 1970). What was not discussed by Becker et al. (2016) was that many specimens originally designated as $S$. cooperi hassi were later synonymized with $S$. isosticha by Klapper \& Phillip (1971). As a result, the name hassi has now been applied to a variety of species in the literature that range nearly the entire Kinderhookian from what was the Upper duplicata Zone to the isosticha-Upper crenulata Zone of Sandberg et al. (1978). It is for this reason that we choose not to use either hassi or jii as a zonal designation but rather the comparatively taxonomically stable species $S$. cooperi as the name-bearer for the zone (see Hogancamp et al. 2019).

\section{Mississippi Valley stratigraphy}

The stratigraphy in the tri-state area is diverse with an abundance of different lithologies and stratigraphic units. The two units of primary interest for the placement of the DCB in the tri-state area are the McCraney Fm., which resides above the current boundary placement in the region and the Louisiana Fm., which lies below the current boundary placement in the region. These units bear a striking resemblance to each other with a unique lithology of gray to brown, sublithographic limestone with silty dolomite partings that break in subconchoidal fractures (Chauffe \& Guzman 1997, Witzke 2002). Due to this striking lithologic similarity and a lack or low abundance of fossils from this facies, the McCraney Fm. and the Louisiana Fm. have been generally correlated based on lithology alone.

The unique facies of the Louisiana and McCraney formations occur at different positions with respect to the Hannibal and Prospect Hill formations (Fig. 4). The Louisiana Formation in Louisiana, MO, and Jersey County, IL, is below the Hannibal, whereas sections containing the McCraney in Newark, MO and the type section in Kinderhook, IL, place the McCraney above the Hannibal. A similar facies relationship to sections in Louisiana, MO and Jersey County, IL, can be seen in Burlington, IA, where the "McCraney" lies below the Prospect Hill, and overlays the uppermost Famennian English River Formation. Witzke (2002) proposed the idea that the "McCraney" at the Starr's Cave outcrop near Burlington, IA was miscorrelated originally by Moore (1928) and then by all subsequent workers (e.g., Witzke et al. 1990, Witzke \& Bunker 2001). Here, we provide new conodont and biostratigraphic evidence for the chronostratigraphic correlation of the "McCraney" in southeast 
Iowa that has significant implications for the placement of the DCB throughout the type Mississippian tri-state area.

\section{Methods}

We sampled the H-28 core and the Starr's Cave outcrop for conodont biostratigraphy and carbonate carbon isotope $\left(\delta^{13} \mathrm{C}_{\mathrm{carb}}\right)$ chemostratigraphy. For chemostratigraphy, the $\mathrm{H}-28$ core was sampled at one foot $(0.30 \mathrm{~m})$ intervals and the Starr's Cave outcrop at $10 \mathrm{~cm}$ intervals using drills fitted with tungsten-carbide tile bits with a preference for fine-grained carbonates (e.g., Saltzman et al. 2002). Samples were analyzed at the Keck Paleoenvironmental and Environmental Stable Isotope Laboratory (KPESIL) at the University of Kansas where powdered sample was

Table 1. Stable isotope data from H-28 Core, Lee County, IA.

\begin{tabular}{|c|c|c|c|c|c|c|c|c|c|}
\hline Decimal Feet & $\delta^{13} \mathrm{C}_{\text {carb }}$ & $\delta^{18} \mathrm{O}$ & Formation & Member & Decimal Feet & $\delta^{13} \mathrm{C}_{\text {carb }}$ & $\delta^{18} \mathrm{O}$ & Formation & Member \\
\hline 153 & 3.91 & -4.11 & Burlington & Cedar Fork & 199 & 2.95 & -4.67 & Burlington & Dolbee Creek \\
\hline 154 & 3.98 & -3.86 & Burlington & Cedar Fork & 200 & 3.14 & -3.07 & Burlington & Dolbee Creek \\
\hline 154.8 & 3.94 & -3.86 & Burlington & Cedar Fork & 201 & 3.05 & -4.23 & Burlington & Dolbee Creek \\
\hline 156 & 3.91 & -4.81 & Burlington & Cedar Fork & 202 & 3.07 & -4.06 & Burlington & Dolbee Creek \\
\hline 156.6 & 3.83 & -5.18 & Burlington & Cedar Fork & 203 & 3.12 & -4.11 & Burlington & Dolbee Creek \\
\hline 158 & 3.45 & -4.49 & Burlington & Cedar Fork & 204 & 3.07 & -4.17 & Burlington & Dolbee Creek \\
\hline 159 & 3.47 & -4.27 & Burlington & Cedar Fork & 205 & 3.11 & -3.97 & Burlington & Dolbee Creek \\
\hline 160 & 3.92 & -5.04 & Burlington & Cedar Fork & 206 & 3.10 & -4.32 & Burlington & Dolbee Creek \\
\hline 162 & 3.74 & -5.24 & Burlington & Haight Creek & 207 & 3.00 & -4.31 & Burlington & Dolbee Creek \\
\hline 163 & 3.86 & -4.86 & Burlington & Haight Creek & 208 & 3.12 & -4.43 & Burlington & Dolbee Creek \\
\hline 164 & 3.66 & -5.48 & Burlington & Haight Creek & 209 & 3.04 & -4.75 & Burlington & Dolbee Creek \\
\hline 165 & 3.58 & -5.46 & Burlington & Haight Creek & 210 & 3.00 & -4.61 & Burlington & Dolbee Creek \\
\hline 166 & 3.77 & -5.11 & Burlington & Haight Creek & 211 & 3.61 & -4.88 & Wassonville & Upper Mb. \\
\hline 167 & 3.74 & -4.87 & Burlington & Haight Creek & 212 & 3.52 & -5.12 & Wassonville & Upper Mb. \\
\hline 168 & 3.73 & -4.78 & Burlington & Haight Creek & 213 & 3.65 & -4.86 & Wassonville & Upper Mb. \\
\hline 169 & 3.67 & -5.21 & Burlington & Haight Creek & 214 & 3.36 & -4.46 & Wassonville & Upper Mb. \\
\hline 171 & 2.86 & -5.63 & Burlington & Haight Creek & 215 & 3.21 & -4.97 & Wassonville & Upper Mb. \\
\hline 172 & 2.96 & -5.19 & Burlington & Haight Creek & 216 & 3.12 & -3.75 & Wassonville & Upper Mb. \\
\hline 173 & 3.45 & -4.66 & Burlington & Haight Creek & 217 & 3.28 & -5.02 & Wassonville & Upper Mb. \\
\hline 173.8 & 3.20 & -4.89 & Burlington & Haight Creek & 218 & 2.93 & -5.52 & Wassonville & Upper Mb. \\
\hline 175 & 3.55 & -4.15 & Burlington & Haight Creek & 219 & 2.59 & -5.86 & Wassonville & Upper Mb. \\
\hline 176 & 3.55 & -4.06 & Burlington & Haight Creek & 220 & 2.62 & -5.83 & Wassonville & Upper Mb. \\
\hline 176.7 & 3.69 & -5.09 & Burlington & Haight Creek & 221 & 2.65 & -5.71 & Wassonville & Upper Mb. \\
\hline 178 & 3.08 & -5.26 & Burlington & Haight Creek & 221.5 & 2.91 & -4.40 & Wassonville & Upper Mb. \\
\hline 179 & 2.97 & -4.81 & Burlington & Haight Creek & 222 & 3.10 & -3.25 & Wassonville & Upper Mb. \\
\hline 180 & 3.00 & -4.85 & Burlington & Haight Creek & 222.5 & 2.51 & -5.80 & Wassonville & Upper Mb. \\
\hline 181 & 2.92 & -5.25 & Burlington & Haight Creek & 223 & 2.78 & -5.63 & Wassonville & Starr's Cave \\
\hline 182 & 3.22 & -4.59 & Burlington & Haight Creek & 223.5 & 2.75 & -5.58 & Wassonville & Starr's Cave \\
\hline 183 & 2.98 & -5.19 & Burlington & Haight Creek & 224 & 2.92 & -5.40 & Wassonville & Starr's Cave \\
\hline 184 & 3.45 & -2.52 & Burlington & Haight Creek & 224.5 & 2.73 & -5.67 & Wassonville & Starr's Cave \\
\hline 184.6 & 3.46 & -3.98 & Burlington & Haight Creek & 224.8 & 2.23 & -5.39 & Wassonville & Starr's Cave \\
\hline 186 & 3.46 & -2.37 & Burlington & Haight Creek & 225 & 2.67 & -1.25 & Wassonville & Starr's Cave \\
\hline 186.8 & 3.44 & -2.16 & Burlington & Haight Creek & 225.5 & 2.49 & -4.82 & Prospect Hill & \\
\hline 188 & 3.43 & -1.91 & Burlington & Haight Creek & 226 & 2.72 & -1.50 & Prospect Hill & \\
\hline 189 & 3.41 & -4.65 & Burlington & Haight Creek & 226.5 & 2.24 & -7.96 & Prospect Hill & \\
\hline 190 & 3.25 & -2.31 & Burlington & Haight Creek & 227 & 2.63 & -2.33 & Prospect Hill & \\
\hline 191 & 3.43 & -2.10 & Burlington & Haight Creek & 228 & 2.41 & -0.88 & Prospect Hill & \\
\hline 192 & 3.42 & -1.52 & Burlington & Haight Creek & 229 & 1.87 & -1.88 & Prospect Hill & \\
\hline 193 & 2.65 & -4.29 & Burlington & Haight Creek & 230 & 1.67 & -2.22 & Prospect Hill & \\
\hline 193.3 & 2.12 & -4.44 & Burlington & Haight Creek & 231 & 3.00 & -1.25 & Prospect Hill & \\
\hline 195 & 2.98 & -4.80 & Burlington & Dolbee Creek & 232 & 2.91 & -1.37 & Prospect Hill & \\
\hline 196 & 2.40 & -4.53 & Burlington & Dolbee Creek & 233 & 2.97 & -1.75 & Prospect Hill & \\
\hline 197 & 2.91 & -5.35 & Burlington & Dolbee Creek & 234 & 3.15 & -1.92 & Prospect Hill & \\
\hline 198 & 2.88 & -4.77 & Burlington & Dolbee Creek & 235 & 2.90 & -2.64 & Prospect Hill & \\
\hline
\end{tabular}


Table 1. Continued.

\begin{tabular}{|c|c|c|c|c|c|c|c|c|c|}
\hline Decimal Feet & $\delta^{13} \mathrm{C}_{\text {carb }}$ & $\delta^{18} \mathrm{O}$ & Formation & Member & Decimal Feet & $\delta^{13} \mathrm{C}_{\text {carb }}$ & $\delta^{18} \mathrm{O}$ & Formation & Member \\
\hline 236 & 2.97 & -2.18 & Prospect Hill & & 289 & 5.62 & -5.41 & Louisiana & \\
\hline 237 & 3.01 & -2.28 & Prospect Hill & & 290 & 5.26 & -5.57 & Louisiana & \\
\hline 238 & 2.91 & -2.70 & Prospect Hill & & 291 & 5.80 & -4.51 & Louisiana & \\
\hline 239 & 2.40 & -4.22 & Prospect Hill & & 292 & 5.73 & -5.13 & Louisiana & \\
\hline 240 & 2.98 & -3.02 & Prospect Hill & & 293 & 5.97 & -4.84 & Louisiana & \\
\hline 241 & 2.92 & -4.35 & Prospect Hill & & 294 & 5.54 & -5.28 & Louisiana & \\
\hline 242 & 1.28 & -4.19 & Prospect Hill & & 295 & 5.60 & -5.33 & Louisiana & \\
\hline 243 & 1.82 & -3.58 & Prospect Hill & & 296 & 5.60 & -5.02 & Louisiana & \\
\hline 244 & 1.09 & -4.28 & Prospect Hill & & 297 & 5.78 & -4.84 & Louisiana & \\
\hline 245 & 2.38 & -4.09 & Prospect Hill & & 298 & 5.67 & -4.68 & Louisiana & \\
\hline 246 & 2.55 & -3.43 & Prospect Hill & & 299 & 5.81 & -4.36 & Louisiana & \\
\hline 247 & 2.51 & -3.30 & Prospect Hill & & 300 & 5.76 & -4.30 & Louisiana & \\
\hline 248 & 2.53 & -4.52 & Prospect Hill & & 301 & 5.55 & -5.24 & Louisiana & \\
\hline 249 & 2.11 & -3.88 & Prospect Hill & & 302 & 5.97 & -4.38 & Louisiana & \\
\hline 250 & 2.21 & -4.41 & Prospect Hill & & 302.8 & 5.46 & -5.52 & Louisiana & \\
\hline 251 & 1.96 & -3.19 & Prospect Hill & & 303.9 & 5.58 & -4.96 & Louisiana & \\
\hline 252 & 2.74 & -2.30 & Prospect Hill & & 304.9 & 5.62 & -5.18 & Louisiana & \\
\hline 253 & 1.34 & -2.94 & Prospect Hill & & 306.2 & 5.87 & -4.65 & Louisiana & \\
\hline 254 & 1.83 & -2.38 & Prospect Hill & & 307 & 5.92 & -4.00 & Louisiana & \\
\hline 255 & 2.70 & -1.48 & Prospect Hill & & 308 & 5.38 & -5.40 & Louisiana & \\
\hline 256 & 3.55 & -1.09 & Prospect Hill & & 309 & 5.83 & -4.68 & Louisiana & \\
\hline 257 & 3.69 & -1.80 & Prospect Hill & & 310 & 5.69 & -4.91 & Louisiana & \\
\hline 257.8 & 4.28 & -3.58 & Louisiana & & 311 & 5.55 & -3.91 & Louisiana & \\
\hline 258 & 4.55 & -4.74 & Louisiana & & 312 & 5.37 & -5.15 & Louisiana & \\
\hline 259 & 4.74 & -4.93 & Louisiana & & 313 & 5.63 & -4.48 & Louisiana & \\
\hline 260 & 4.64 & -5.14 & Louisiana & & 314 & 5.65 & -4.46 & Louisiana & \\
\hline 260.9 & 4.66 & -4.89 & Louisiana & & 315 & 5.31 & -4.33 & Louisiana & \\
\hline 262 & 5.04 & -4.76 & Louisiana & & 316 & 5.43 & -4.60 & Louisiana & \\
\hline 263 & 5.00 & -5.09 & Louisiana & & 317 & 5.20 & -4.44 & Louisiana & \\
\hline 264 & 5.49 & -4.76 & Louisiana & & 318 & 5.28 & -4.26 & Louisiana & \\
\hline 265 & 4.98 & -4.89 & Louisiana & & 319 & 5.21 & -4.28 & Louisiana & \\
\hline 266 & 5.47 & -4.87 & Louisiana & & 320 & 5.06 & -4.13 & Louisiana & \\
\hline 267 & 5.53 & -4.79 & Louisiana & & 321 & 4.91 & -4.24 & Louisiana & \\
\hline 268 & 5.73 & -4.84 & Louisiana & & 322 & 4.60 & -4.16 & Louisiana & \\
\hline 269 & 5.71 & -4.91 & Louisiana & & 323 & 3.18 & -4.49 & Louisiana & \\
\hline 270 & 5.40 & -5.15 & Louisiana & & 324 & 2.67 & -3.72 & Louisiana & \\
\hline 271 & 5.94 & -4.87 & Louisiana & & 325 & 3.09 & -2.80 & Louisiana & \\
\hline 272 & 5.27 & -4.31 & Louisiana & & 326 & 1.92 & -2.41 & English River & \\
\hline 273 & 5.48 & -4.60 & Louisiana & & 327 & 2.29 & -2.13 & English River & \\
\hline 274 & 6.00 & -4.98 & Louisiana & & 328 & 2.01 & -2.05 & English River & \\
\hline 275 & 5.65 & -5.12 & Louisiana & & 329 & 1.24 & -2.71 & English River & \\
\hline 276 & 5.82 & -4.99 & Louisiana & & 330 & 1.58 & -1.95 & English River & \\
\hline 277 & 5.84 & -4.87 & Louisiana & & 331 & 1.72 & -2.00 & English River & \\
\hline 278 & 5.86 & -4.94 & Louisiana & & 332 & 1.70 & -2.01 & English River & \\
\hline 279 & 5.82 & -4.52 & Louisiana & & 333.2 & 1.46 & -2.41 & English River & \\
\hline 280 & 5.79 & -4.12 & Louisiana & & 334 & 1.89 & -1.86 & English River & \\
\hline 281 & 5.76 & -5.03 & Louisiana & & 335 & 1.73 & -2.36 & English River & \\
\hline 282 & 5.65 & -5.14 & Louisiana & & 336 & 0.21 & -3.66 & English River & \\
\hline 283 & 5.61 & -5.23 & Louisiana & & 337 & -0.24 & -4.47 & English River & \\
\hline 284 & 5.63 & -5.34 & Louisiana & & 338 & 0.28 & -3.58 & English River & \\
\hline 285 & 5.95 & -4.36 & Louisiana & & 339 & 0.54 & -3.77 & English River & \\
\hline 286 & 5.65 & -5.13 & Louisiana & & 340 & 1.03 & -2.82 & English River & \\
\hline 287 & 5.67 & -4.90 & Louisiana & & 340.6 & 0.45 & -3.67 & English River & \\
\hline 288 & 5.42 & -5.57 & Louisiana & & 341.9 & 0.85 & -1.76 & English River & \\
\hline
\end{tabular}


Table 2. Stable isotope data from Starr's Cave, Burlington, IA.

\begin{tabular}{|c|c|c|c|c|}
\hline Meters & $\delta^{13} \mathrm{C}_{\text {carb }}$ & $\delta^{18} \mathrm{O}$ & Formation & Member \\
\hline 7.0 & 1.57 & -1.84 & Wassonville & Upper Mb. \\
\hline 6.9 & 1.12 & -2.02 & Wassonville & Upper Mb. \\
\hline 6.8 & 0.20 & -4.98 & Wassonville & Upper Mb. \\
\hline 6.7 & 0.55 & -1.93 & Wassonville & Upper Mb. \\
\hline 6.6 & 2.08 & -0.93 & Wassonville & Upper Mb. \\
\hline 6.5 & 1.61 & -2.60 & Wassonville & Upper Mb. \\
\hline 6.4 & 0.98 & -4.86 & Wassonville & Upper Mb. \\
\hline 6.3 & 0.75 & -4.43 & Wassonville & Upper Mb. \\
\hline 6.2 & 1.10 & -4.34 & Wassonville & Starr's Cave \\
\hline 6.1 & 0.42 & -5.25 & Wassonville & Starr's Cave \\
\hline 6.0 & 0.85 & -4.54 & Wassonville & Starr's Cave \\
\hline 5.9 & 1.16 & -4.36 & Wassonville & Starr's Cave \\
\hline 5.8 & 0.87 & -4.70 & Wassonville & Starr's Cave \\
\hline 5.7 & 1.38 & -3.89 & Wassonville & Starr's Cave \\
\hline 5.6 & 1.21 & -4.16 & Wassonville & Starr's Cave \\
\hline 5.5 & 0.93 & -4.57 & Wassonville & Starr's Cave \\
\hline 5.4 & 2.52 & -0.80 & Prospect Hill & \\
\hline 5.3 & 2.24 & -1.42 & Prospect Hill & \\
\hline 5.2 & 1.57 & -2.41 & Prospect Hill & \\
\hline 5.1 & 1.48 & -1.77 & Prospect Hill & \\
\hline 5.0 & 1.34 & -2.04 & Prospect Hill & \\
\hline 4.9 & 2.02 & -1.18 & Prospect Hill & \\
\hline 4.8 & 1.52 & -1.50 & Prospect Hill & \\
\hline 4.7 & 1.64 & -2.71 & Prospect Hill & \\
\hline 4.6 & 1.52 & -1.76 & Prospect Hill & \\
\hline 4.5 & 1.16 & -1.41 & Prospect Hill & \\
\hline 4.4 & 1.48 & -1.29 & Prospect Hill & \\
\hline 4.3 & 1.90 & -1.10 & Prospect Hill & \\
\hline 4.2 & -0.16 & -2.57 & Prospect Hill & \\
\hline 4.1 & 3.41 & -0.51 & Prospect Hill & \\
\hline 4.0 & -0.05 & -2.20 & Prospect Hill & \\
\hline 3.5 & 4.71 & -0.03 & “McCraney" & \\
\hline 3.4 & 1.04 & -3.63 & “McCraney” & \\
\hline 3.3 & 4.92 & 0.46 & “McCraney" & \\
\hline 3.2 & 2.73 & -1.20 & “McCraney" & \\
\hline 3.1 & 3.70 & -0.37 & “McCraney" & \\
\hline 3.0 & 1.64 & -4.34 & "McCraney" & \\
\hline 2.9 & 1.73 & -4.40 & "McCraney" & \\
\hline 2.8 & 2.17 & -4.61 & "McCraney" & \\
\hline 2.7 & 2.35 & -4.55 & "McCraney" & \\
\hline 2.6 & 2.11 & -4.64 & "McCraney" & \\
\hline
\end{tabular}

reacted with $100 \%$ phosphoric acid with density $>1.9$ (Wachter Hayes 1985) with a KIEL Carbonate Device connected to a ThermoFinnigan MAT 253 isotope ratio mass spectrometer. Isotopic values were calibrated to VPDB using NBS-18 and NBS-19 as primary standards, and daily performance was monitored with laboratory (secondary) standards TSF-1, SIGMA CALCITE, and 88b

\begin{tabular}{|c|c|c|c|c|}
\hline Meters & $\delta^{13} \mathrm{C}_{\text {carb }}$ & $\delta^{18} \mathrm{O}$ & Formation & Member \\
\hline 2.5 & 2.15 & -4.50 & "McCraney" & \\
\hline 2.4 & 2.26 & -4.74 & "McCraney" & \\
\hline 2.3 & 2.05 & -4.70 & "McCraney" & \\
\hline 2.2 & 2.35 & -4.58 & "McCraney" & \\
\hline 2.1 & 2.52 & -4.51 & "McCraney" & \\
\hline 2.0 & 2.46 & -4.57 & "McCraney" & \\
\hline 1.9 & 2.51 & -4.65 & "McCraney" & \\
\hline 1.8 & 2.19 & -4.67 & "McCraney" & \\
\hline 1.7 & 2.23 & -4.59 & "McCraney" & \\
\hline 1.6 & 2.80 & -4.44 & "McCraney" & \\
\hline 1.5 & 2.87 & -4.51 & "McCraney" & \\
\hline 1.4 & 1.83 & -4.16 & "McCraney" & \\
\hline 1.3 & 2.79 & -4.39 & "McCraney" & \\
\hline 1.2 & 3.27 & -4.62 & "McCraney" & \\
\hline 1.1 & 2.81 & -4.42 & "McCraney" & \\
\hline 1.0 & 3.02 & -4.44 & "McCraney" & \\
\hline 0.9 & 3.46 & -4.42 & "McCraney" & \\
\hline 0.8 & 3.13 & -4.49 & "McCraney" & \\
\hline 0.7 & 3.10 & -4.52 & "McCraney" & \\
\hline 0.6 & 3.56 & -4.56 & "McCraney" & \\
\hline 0.5 & 2.38 & -4.75 & "McCraney" & \\
\hline 0.4 & 2.63 & -4.38 & "McCraney" & \\
\hline 0.3 & 2.16 & -4.27 & "McCraney" & \\
\hline 0.2 & 2.32 & -4.70 & "McCraney" & \\
\hline 0.1 & -0.02 & -12.45 & "McCraney" & \\
\hline 0.0 & 1.19 & -5.00 & English River & \\
\hline-0.1 & 1.62 & -1.80 & English River & \\
\hline-0.2 & 1.55 & -1.88 & English River & \\
\hline-0.3 & 2.48 & -0.96 & English River & \\
\hline-0.4 & 1.63 & -1.63 & English River & \\
\hline-0.5 & 2.17 & -0.82 & English River & \\
\hline-0.6 & 1.85 & -1.06 & English River & \\
\hline-0.7 & 2.00 & -0.81 & English River & \\
\hline-0.8 & 2.08 & -0.92 & English River & \\
\hline-0.9 & 2.06 & -0.84 & English River & \\
\hline-1.0 & 2.04 & -0.90 & English River & \\
\hline-1.1 & 1.81 & -0.96 & English River & \\
\hline-1.2 & 0.94 & -1.00 & English River & \\
\hline-1.3 & -0.39 & -3.05 & English River & \\
\hline-1.4 & 1.23 & -0.62 & English River & \\
\hline-1.5 & 0.60 & -2.56 & English River & \\
\hline
\end{tabular}

Dolomite analyzed at the beginning, middle, and end of each 40 sample queue. Precision was better than $0.10 \%$ for both carbon and oxygen isotopes.

Both the H-28 Core and the Starr's Cave outcrop were sampled for conodont biostratigraphy. Continuous samples (50 total) were taken every foot $(0.30 \mathrm{~m})$ to as small as every three inches $(7.5 \mathrm{~cm})$ from the $\mathrm{H}-28$ Core,

Figure 5. Conodont biostratigraphy and carbon isotope chemostratigraphy of the H-28 core from Burlington, Iowa. Conodont occurrences are represented by small circles. The red dashed line is a proposed position of the Devonian-Carboniferous boundary at the base of the Protognathodus kockeli Zone. Note the use of Louisiana Fm. in the core. Previously this strata in Iowa had been referred to as "McCraney". 


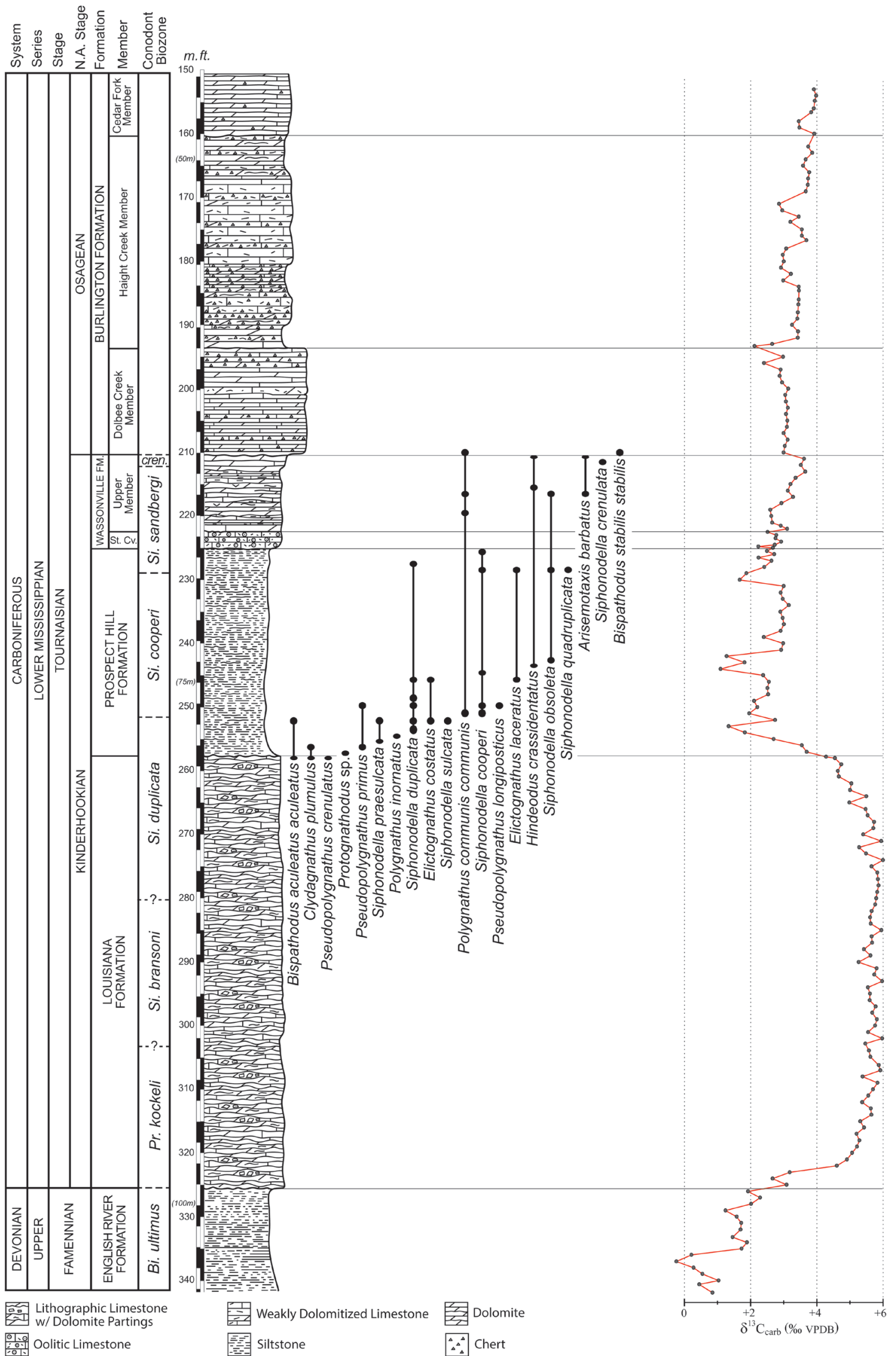


from the top of the Louisiana Limestone to the top of the Wassonville Fm. sampling the entire core over this interval. Not all samples yielded conodonts, and those that did yield identifiable specimens are shown in Fig. 5. The Starr's Cave outcrop was similarly sampled at $30-40 \mathrm{~cm}$ resolution. Conodont samples were dissolved in a $10 \%$ double-buffered formic acid solution (Jeppsson \& Anehus 1995). Residues were sieved through 1,000 and 63 micron sieves, and the 63-micron fraction was subjected to heavy liquid separation using lithium metatungstate at $2.83-2.84 \mathrm{~g} / \mathrm{mL}$. Conodonts were then picked under a microscope and photographed using a Cannon 60D camera with a StackShot platform and Zerene imagestacking software for regular-light images before being gold coated and imaged in a Hitachi S-3400N Scanning Electron Microscope. All conodont samples illustrated here, as well as additional material not imaged, are housed at the University of Iowa Paleontology Repository with accession numbers SUI 148219-SUI 148368.

\section{Results}

\section{H-28 Core}

The H-28 core (Iowa Geological Survey WNumber 27539 ) is located in southeastern Iowa, south of the city of Burlington, at $40.408131^{\circ} \mathrm{N}, 91.417870^{\circ} \mathrm{W}$. The sampled section spans 190 feet $(58 \mathrm{~m})$ and contains the English River, Louisiana, Prospect Hill, Wassonville, and Burlington formations. Two hundred seventeen carbon isotope samples were taken at mostly one-foot intervals $(0.30 \mathrm{~m})$ and 50 conodont samples were taken from the top of the Louisiana through Wassonville formations every 12 inches $(30 \mathrm{~cm})$ with some samples every three inches $(7.5 \mathrm{~cm})$ for continuous sampling of the entire core in this interval. Carbonate carbon isotope $\left(\delta^{13} \mathrm{C}_{\text {carb }}\right)$ values ranged from $-0.05 \%$ to $+6.0 \%$ (Fig. 5) and the data are presented in Tab. 1. This includes the Hangenberg positive isotope excursion, which occurs from the upper English River Fm. to the lowermost Prospect Hill Fm. Peak values of $+6 \%$ occur throughout the Louisiana Formation. Conodont samples returned a diverse Siphonodella fauna. Biostratigraphically important specimens include Siphonodella praesulcata, S. sulcata, S. duplicata, S. cooperi, S. obsoleta, and Hindeodus crassidentatus in the Prospect Hill Fm. and S. quadruplicata, Arisemotaxis barbatus, and S. crenulata in the Wassonville Formation (Figs 6-10, 12).

\section{Starr's Cave}

The Starr's Cave locality is located in Starr's Cave Park and Preserve, north of the city of Burlington, Des Moines
County, at $40.851537^{\circ} \mathrm{N}, 91.136000^{\circ} \mathrm{W}$ (Fig. 11). The section was sampled from the base of the English River Fm. into the Upper Member of the Wassonville Fm. Conodont samples were taken at roughly $30-40 \mathrm{~cm}$ intervals and 82 carbon isotope samples were taken at $10 \mathrm{~cm}$ resolution. Carbonate carbon isotope $\left(\delta^{13} \mathrm{C}_{\text {carb }}\right)$ data ranged from $-0.5 \%$ to $+5 \%$ with peak values occurring near the top of the "McCraney" Fm. (Fig. 13) and the data are presented in Tab. 2. Conodont samples returned a diverse conodont fauna many of which are from the Prospect Hill Fm. Biostratigraphically important specimens include Siphonodella duplicata, S. quadruplicata, S. cooperi, S. sulcata, S. obsoleta, and S. sandbergi (Figs 14-17).

\section{Discussion}

The data recovered by this study provide important new information regarding the distinction between the Louisiana and McCraney formations in southeastern Iowa. Below we summarize the chronostratigraphic information available for these units in their type areas and compare them to the new data provided from southeastern Iowa.

Conodont biostratigraphic and chemostratigraphic data unequivocally demonstrate the chronostratigraphic position of the Louisiana Formation in its type area. Conodonts from the Louisiana in its type area include Protognathodus kockeli, P. collinsoni, P. meischneri, and Cryptotaxis culminidirecta (Scott \& Collinson 1961, Straka 1968, Chauffe \& Nichols 1995). Several of these species were originally identified as belonging to the genus Gnathodus and these misidentifications suggested an earliest Mississippian (Kinderhookian) origin for the genus that was not supported anywhere else in the world. To clarify these identifications, we re-illustrate the specimens in question here in Fig. 18. Scott \& Collinson (1961) provided a species list of specimens recovered from the Louisiana Limestone that included Gnathodus cf. G. commutatus (Scott \& Collinson 1961, p. 113). Later in the plate caption to their plate 1 (p. 137) they refer two specimens to Gnathodus commutatus, shown in our Fig. 18 with their original figure designation as image 23,25 , and 26 . These two specimens clearly belong to two species of Protognathodus, with 23 and 25 reidentified herein as oral and basal views of $P$. collinsoni, and image 26 re-identified herein as $P$. meischneri. Image 28 was originally identified as belonging to Gnathodus kockeli, now P. kockeli (see Corradini et al. 2011). All of these specimens are from the Louisiana Limestone at Teneriffe School, Jersey County, Illinois. Straka (1968) also reported a single Kinderhookian occurrence of Gnathodus in Iowa. His specimen, from the Wassonville Formation at the Maple Mill Locality, Washington 


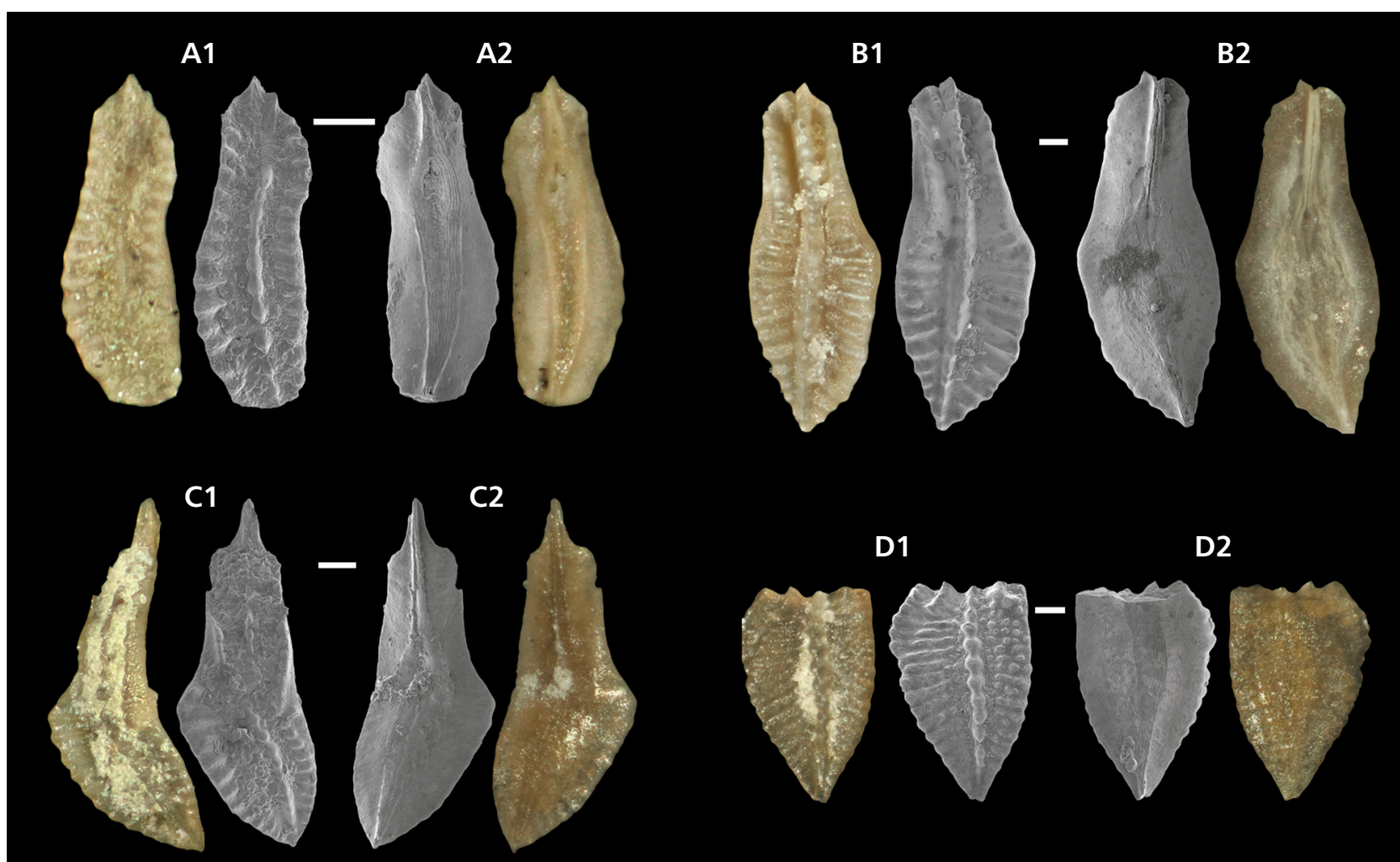

D1

D2
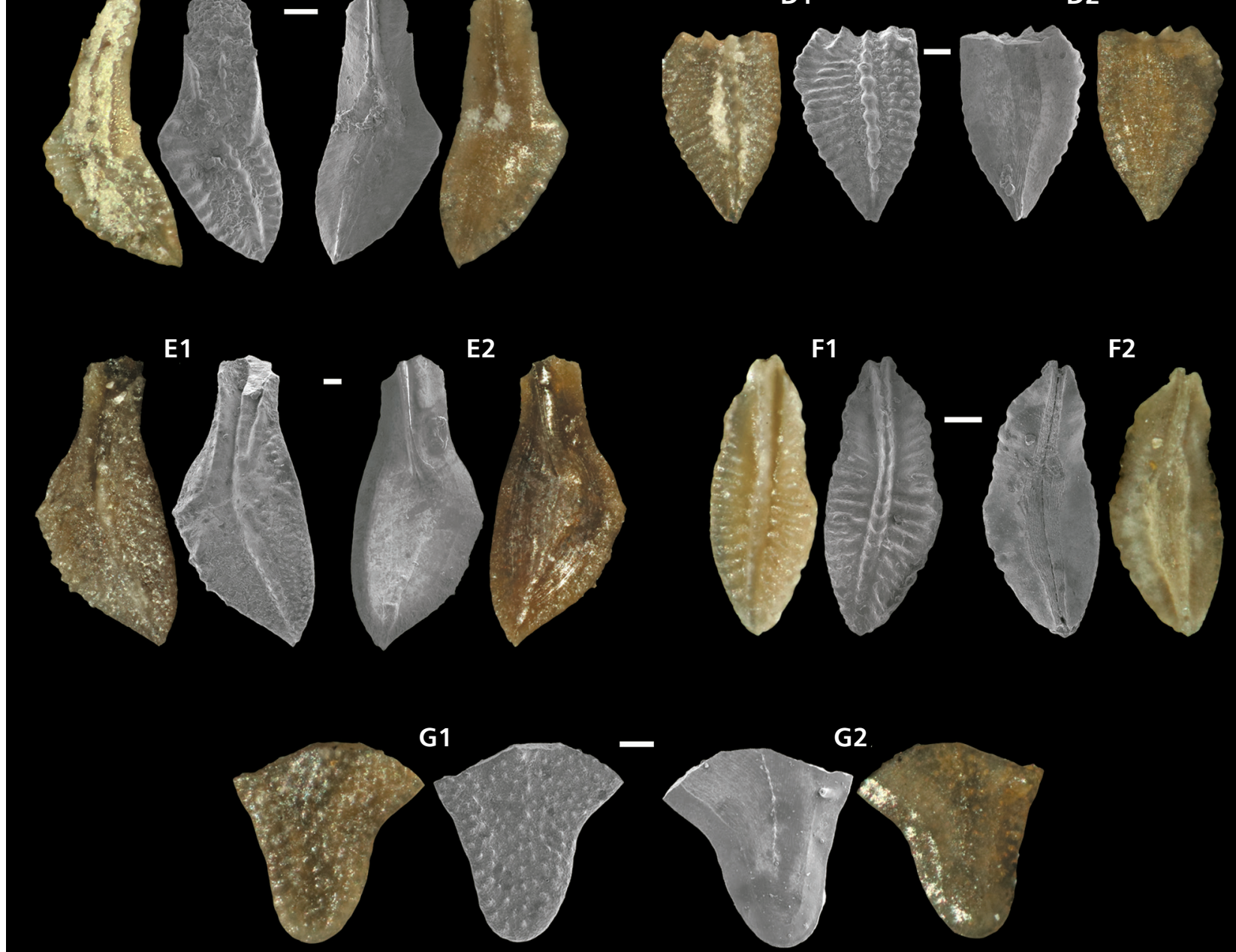

Figure 7. Conodonts from the H-28 Core. - A - Siphonodella praesulcata Sandberg et al., 1972; sinistral $\mathrm{P}_{1}$ element, oral view (A1), aboral view (A2), Prospect Hill Fm., 255'0"-255'9”, SUI 148226. • B, C - Siphonodella duplicata (Branson \& Mehl, 1934a); B - dextral P 1 element, oral view (B1), aboral view (B2), Prospect Hill Fm., 249'2"-250'2”, SUI 148227; C - sinistral P1 element, oral view (C1), aboral view (C2), Prospect Hill Fm., 245'2"-246'1", SUI 148228. • D - Siphonodella ?quadruplicata (Branson \& Mehl, 1934a); sinistral $\mathrm{P}_{1}$ element, oral view (D1), aboral view (D2), Prospect Hill Fm., 228'0”-229’0”, SUI 148229. • E - Siphonodella obsoleta Hass, 1959; sinistral $P_{1}$ element, oral view (E1), aboral view (E2), Prospect Hill Fm., 242'1"-243'1", SUI 148230. • F - Siphonodella sulcata (Huddle, 1934); sinistral $P_{1}$ element, oral view (F1), aboral view (F2), Prospect Hill Fm., 251'7"-252'10", SUI 14823. • G - gen. et sp. indet.; ? $P_{1}$ element, oral view (G1), aboral view (G2), Upper Member, Wassonville Fm., 212'0"-213'0”, SUI 148232. 


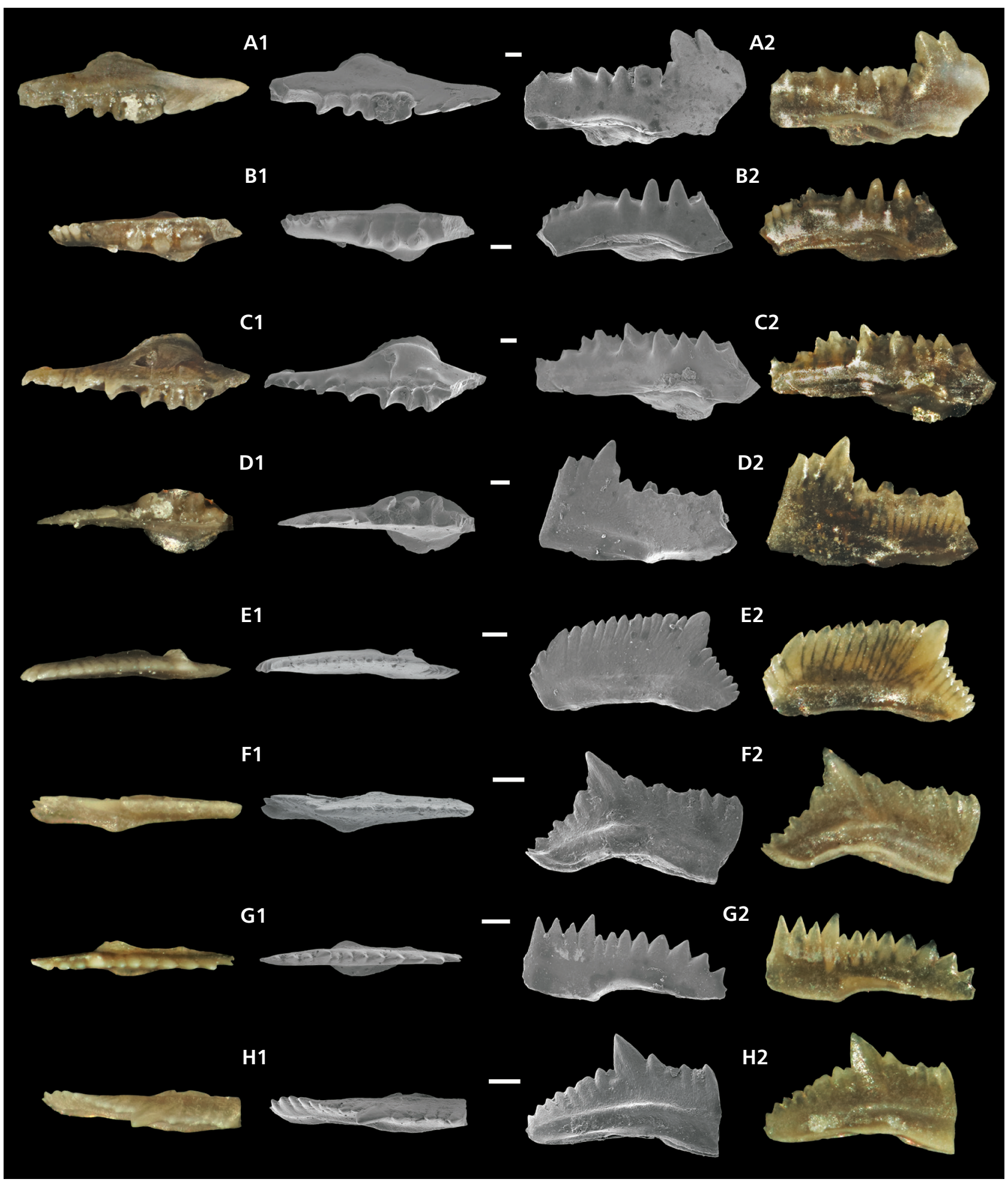

Figure 8. Conodonts from the H-28 Core. A , D - Clydagnathus plumulus (Rhodes et al., 1969); A - sinistral $\mathrm{P}_{1}$ element, oral view (A1), caudal view (A2), Louisiana Fm., 257'8'-258'2", SUI 148233; D - sinistral P1 element, oral view (D1), rostral view (D2), Prospect Hill Fm., 255'9”-256'10", SUI 148234. - B, C - Bispathodus aculeatus aculeatus (Branson \& Meh1, 1934b); B - sinistral $\mathrm{P}_{1}$ element, oral view (B1), caudal view (B2), Louisiana Fm., 257' '"-258'2", SUI 148235; C - sinistral $P_{1}$ element, oral view (C1), caudal view (C2), Louisiana Fm., 257'8"'-258'2", SUI 148236. • E - Elictognathus costatus (Branson \& Mehl, 1934a); E - dextral $\mathrm{P}_{2}$ element, oral view (E1), caudal view (E2), Prospect Hill Fm., 245'2"'-246'1”, SUI 148237. • F, H - Elictognathus laceratus (Branson \& Mehl, 1934a); F - dextral $\mathrm{P}_{2}$ element, oral view (F1), rostral view (F2), Prospect Hill Fm., 245'2"-246'1", SUI 148238; H - dextral $\mathrm{P}_{2}$ element, oral view (H1), rostral view (H2), Prospect Hill Fm., 228'0"'-229'0”, SUI 148239. • G - Hindeodus crassidentatus (Branson \& Mehl, 1934a), dextral $\mathrm{P}_{2}$ element, oral view (G1), caudal view (G2), Prospect Hill Fm., 243'1'”-243'9”, SUI 148240 


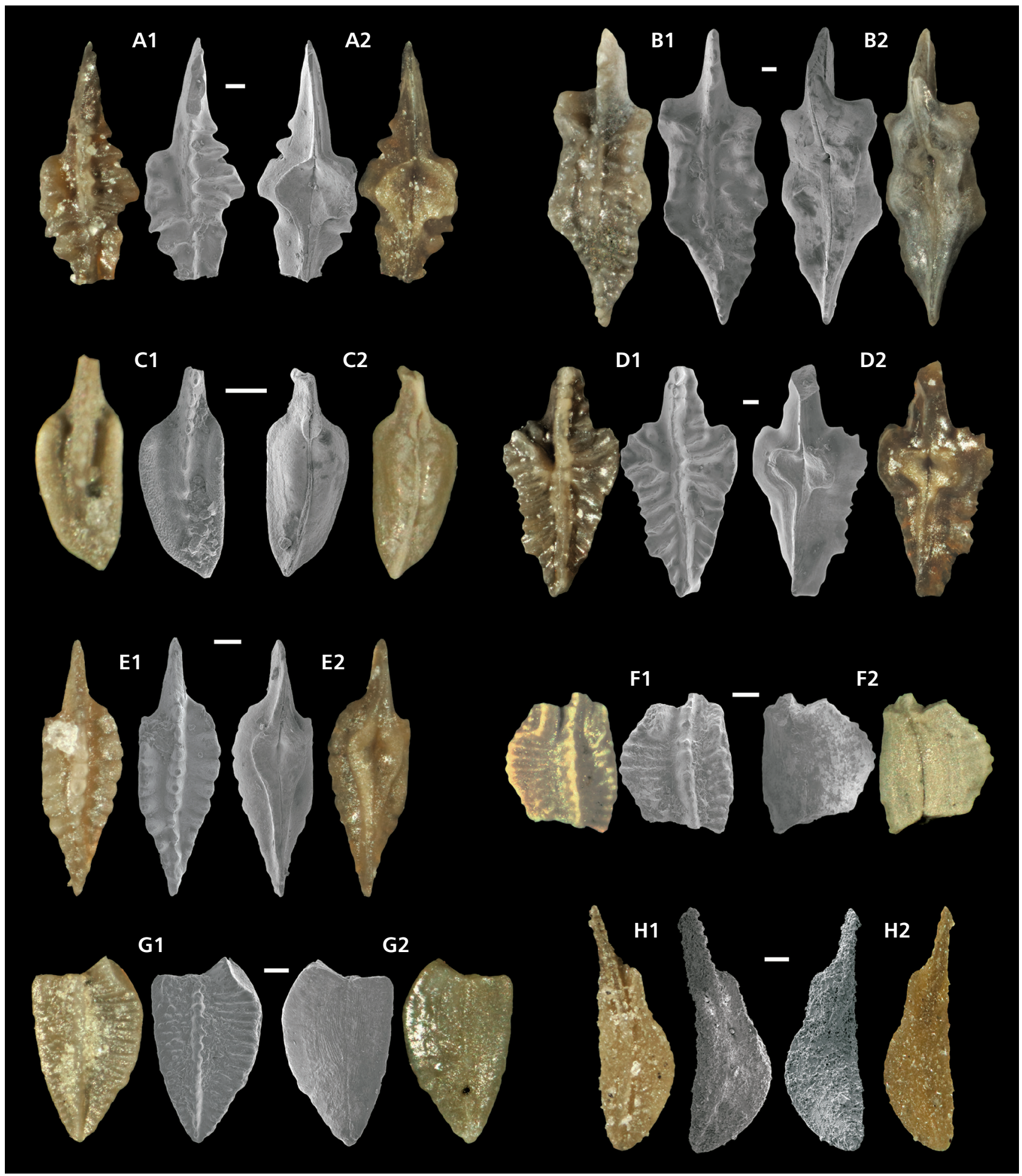

Figure 9. Conodonts from the H-28 Core. A, D - Pseudopolygnathus primus Branson \& Mehl, 1934a; A - sinistral $\mathrm{P}_{1}$ element, oral view (A1), aboral view (A2), Prospect Hill Fm., 255'9"'-256'10", SUI 148241; D - sinistral P 1 element, oral view (D1), aboral view (D2), Prospect Hill Fm., 249'2"-250'2", SUI 148242. • B - Pseudopolygnathus crenulatus Branson \& Mehl, 1934a; sinistral $\mathrm{P}_{1}$ element, oral view (B1), aboral view (B2), Louisiana Fm., 257'8"-258'2", SUI 148243. • C - Polygnathus communis communis (Branson \& Mehl, 1934a); sinistral $\mathrm{P}_{1}$ element, oral view (C1), aboral view (C2), Prospect Hill Fm., 250'2"-251'7', SUI 148244. • E - Polygnathus longiposticus Branson \& Mehl, 1934a; extral $\mathrm{P}_{1}$ element, oral view (E1), aboral view (E2), Prospect Hill Fm., 249'2"-250'2", SUI 148245. • F - Siphonodella sp., sinistral $\mathrm{P}_{1}$ element, oral view (F1), aboral view (F2), Prospect Hill Fm., 241'5"-242’0", SUI 148246. - G - Siphonodella cooperi Hass, 1959, dextral P 1 element, oral view (G1), aboral view (G2), Prospect Hill Fm., 228'0"-229'0”, SUI 148247. • H - Siphonodella sp. - dextral $\mathrm{P}_{1}$ element, oral view (H1), aboral view (H2), Prospect Hill Fm., 220'0"-221'0", SUI 148248. 


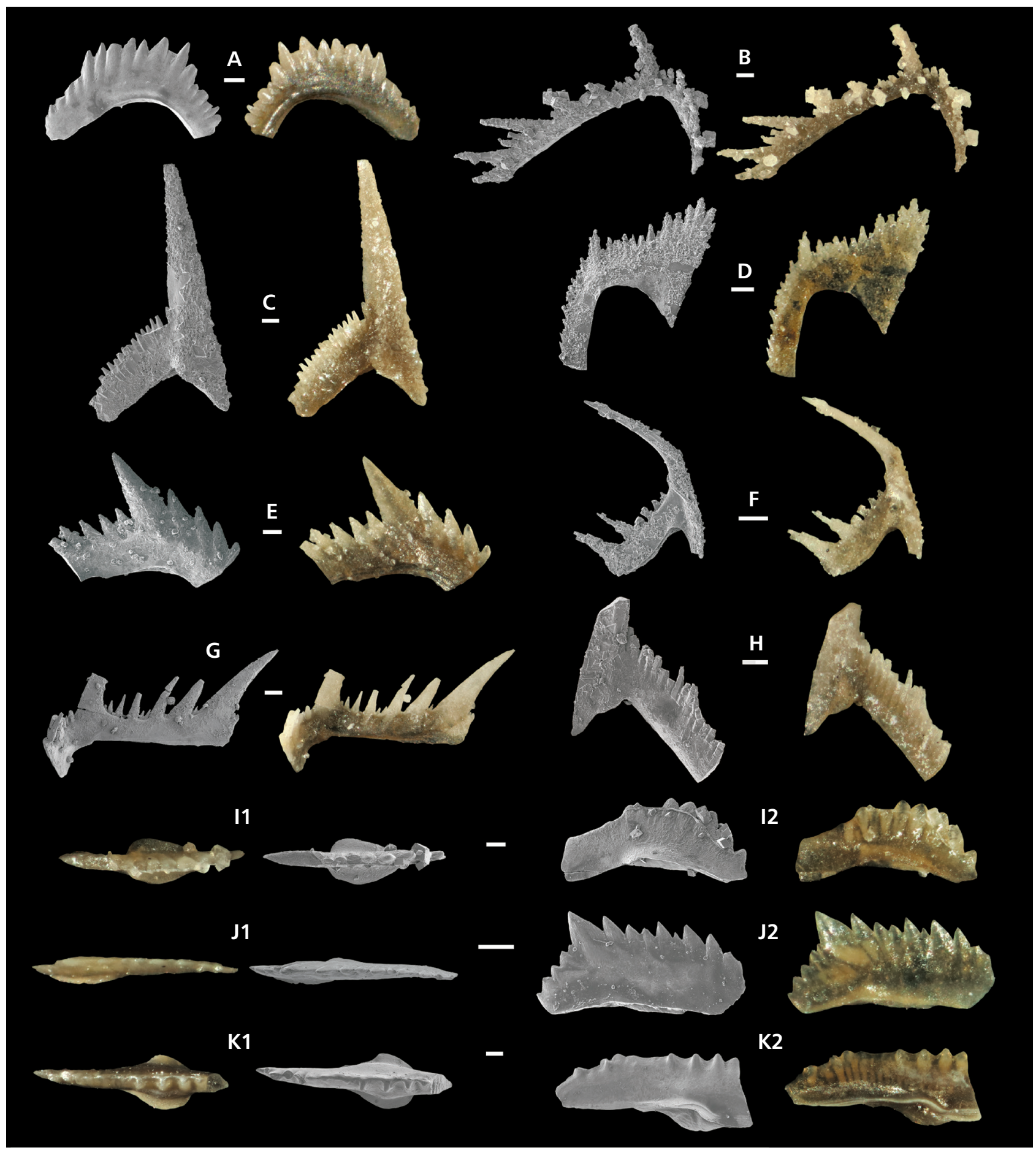

Figure 10. Conodonts from the H-28 Core. • A - Protognathodus sp., sinistral $\mathrm{P}_{2}$ element, rostral view on left (SEM), caudal view on right (plain light), Prospect Hill Fm., 256'10"-257'8", SUI 148249. • B - gen. et sp. indet., sinistral ?S 3 element, adaxial view, Upper Member, Wassonville Fm., 216'0"-217'0", SUI 148250. • C, H - Arisemotaxis barbatus (Branson \& Mehl, 1934a); C - dextral M element, dorsal view, Upper Member, Wassonville Fm., 216'0"-217'0", SUI 148251; H - sinistral M element, dorsal view, Upper Member, Wassonville Fm., 210'5”-211'0”, SUI 148252. • $\mathrm{D}$ - Siphonodella sp., sinistral $\mathrm{S}_{2}$ element, adaxial view, Upper Member, Wassonville Fm., 216'0"-217'0", SUI 148253. • E-G - Hindeodus crassidentatus (Branson \& Mehl, 1934a); E - dextral $\mathrm{P}_{2}$ element, caudal view, Upper Member, Wassonville Fm., 210'5"'-211'0", SUI 148254; F dextral $\mathrm{S}_{2}$ element, adaxial view, Upper Member, Wassonville Fm., 215'0"-216'0”, SUI 148255; G - dextral S element, adaxial view, Upper Member, Wassonville Fm., 210'5"-211'0", SUI 148256. • I - Bispathodus stabilis stabilis (Branson \& Mehl, 1934b), sinistral $\mathrm{P}_{1}$ element, oral view (I1), rostral view (I2), Dolbee Creek Member, Burlington Fm., 209'6"-210'5", SUI 148257. • J - Elictognathus costatus (Branson \& Mehl, 1934a), sinistral $\mathrm{P}_{2}$ element, oral view (J1), caudal view (J2), Prospect Hill Fm., 251'7'-252'10”, SUI 148258. $・ \mathrm{~K}-$ Bispathodus aculeatus aculeatus (Branson \& Mehl, 1934b), sinistral $P_{1}$ element, oral view (K1), caudal view (K2), Prospect Hill Fm., 251'7'-252'10", SUI 148259. 
Figure 11. Field photograph of the Starr's Cave Outcrop at Starr's Cave Park in Des Moines County, IA. Formations and unit members are labeled. The red dashed lines indicate the boundary between members or formations.

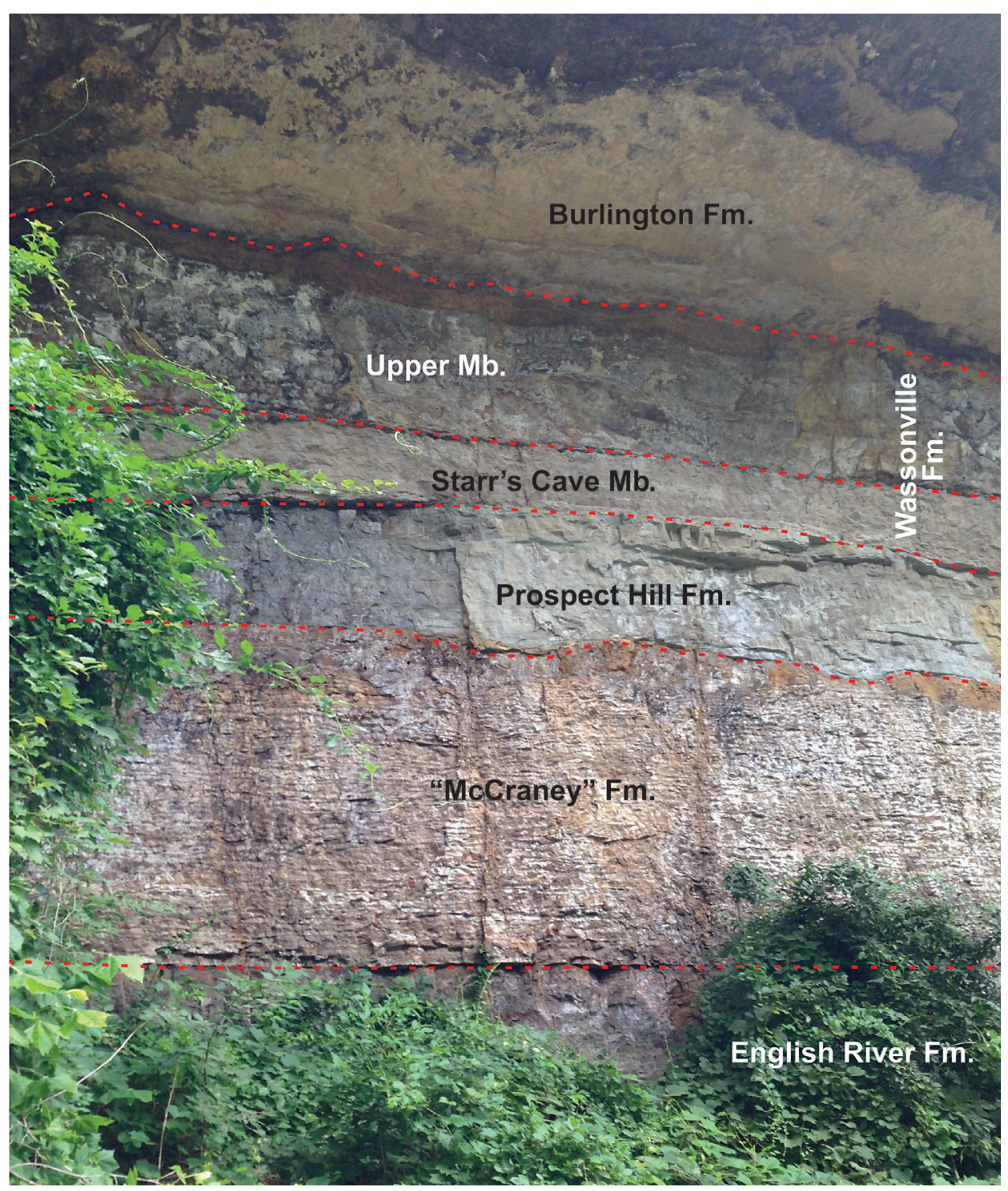

County, Iowa, was originally identified as Gnathodus delicatus. This specimen (spec. 11, pl. 7, Straka 1968) is housed at the University of Iowa Paleontology Repository as SUI \#125484 and was re-examined by the authors. The specimen clearly has a single node on each side of the platform and is best identified as P. collinsoni. Taken together, these re-identifications of reports of Gnathodus in Kinderhookian strata of the tri-state area, and the data presented from the Louisiana Limestone by Chauffe \& Nichols (1995), demonstrate that the entire succession of Protognathodus, including the base of the P. kockeli Zone, occurs within the DCB interval in the tri-state area and that the first occurrence of $P$. kockeli is no lower than the base of the Louisiana Limestone.

Figure 12. Conodonts from the H-28 Core. $-\mathrm{A}, \mathrm{C}-\mathrm{E}-$ Polygnathus communis communis (Branson \& Mehl, 1934a); $\mathrm{A}-$ sinistral $\mathrm{P}_{1}$ element, oral view (A1), aboral view (A2), Upper Member, Wassonville Fm., 219'0"-220'0", SUI 148260; C - sinistral $\mathrm{P}_{1}$ element, oral view (C1), aboral view (C2), Dolbee Creek Member, Burlington Fm., 209'6"-210'5”, SUI 148261; D - dextral $\mathrm{P}_{1}$ element, oral view (D1), aboral view (D2), Upper Member, Wassonville Fm., 216'0"-217'0", SUI 148262; E - sinistral $\mathrm{P}_{1}$ element, oral view (E1), aboral view (E2), Dolbee Creek Member, Burlington Fm., 209'6"-210'5", SUI 148263. • B - Siphonodella sp., dextral P1 element, oral view (B1), aboral view (B2), Upper Member, Wassonville Fm., 216'0"'217'0”, SUI 148264. • F - Siphonodella ?cooperi Hass, 1959; sinistral $P_{1}$ element, oral view (F1), aboral view (F2), Prospect Hill Fm., 244'3"'-245'0", SUI 148265. • G - Siphonodella duplicata (Branson \& Mehl, 1934a); sinistral $P_{1}$ element, oral view (G1), aboral view (G2), Prospect Hill Fm., 227'0"-228'0", SUI 148266. • H - Polygnathus inornatus E.R. Branson, 1934; dextral $\mathrm{P}_{1}$ element, aboral view (H1), oral view (H2), Prospect Hill Fm., 254'3"'-255'0", SUI 148267. I - Siphonodella obsoleta Hass, 1959, sinistral $\mathrm{P}_{1}$ element, aboral view (I1), oral view (I2), Prospect Hill Fm., 227'0"-228'0", SUI 148268. 


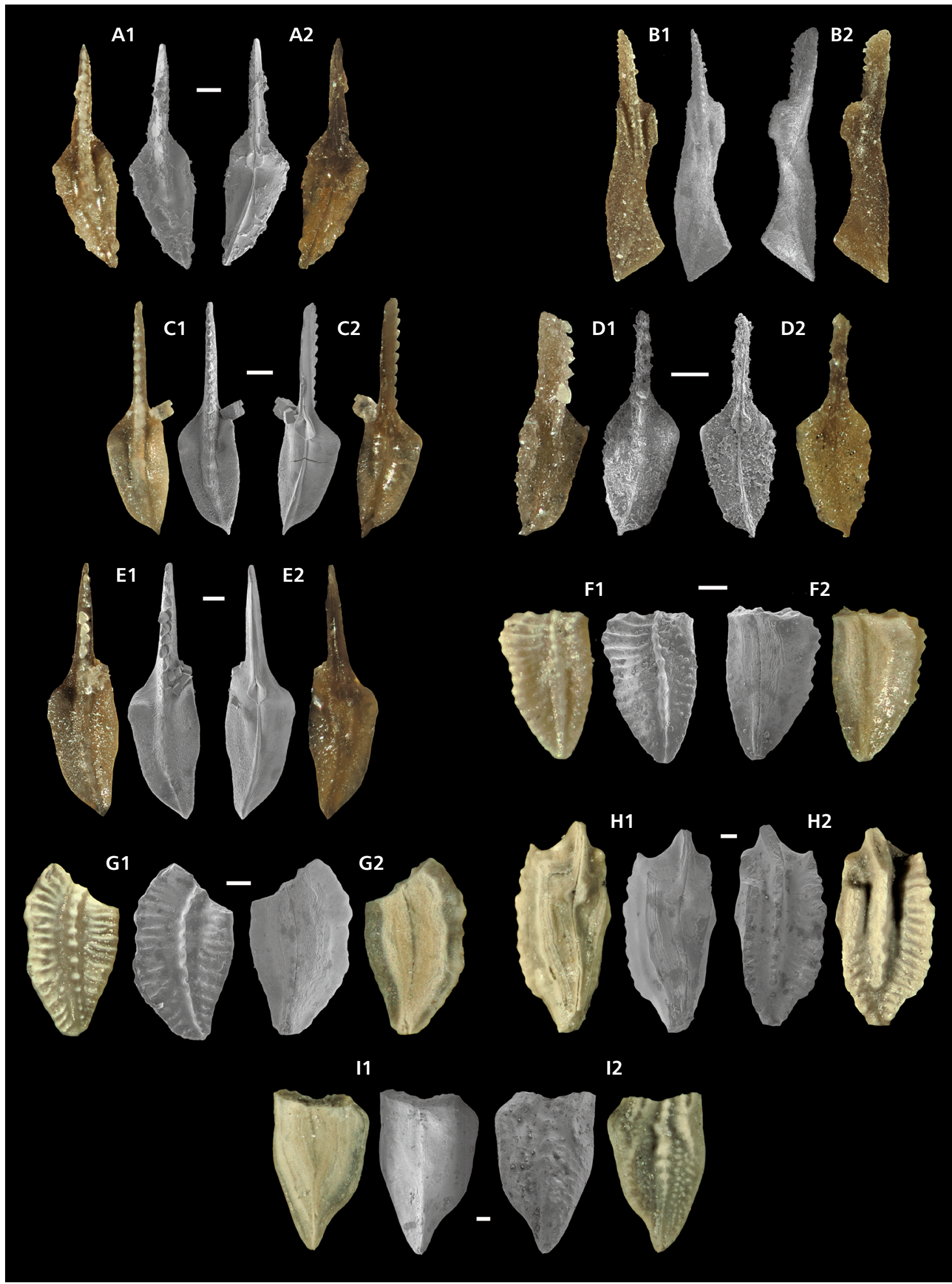




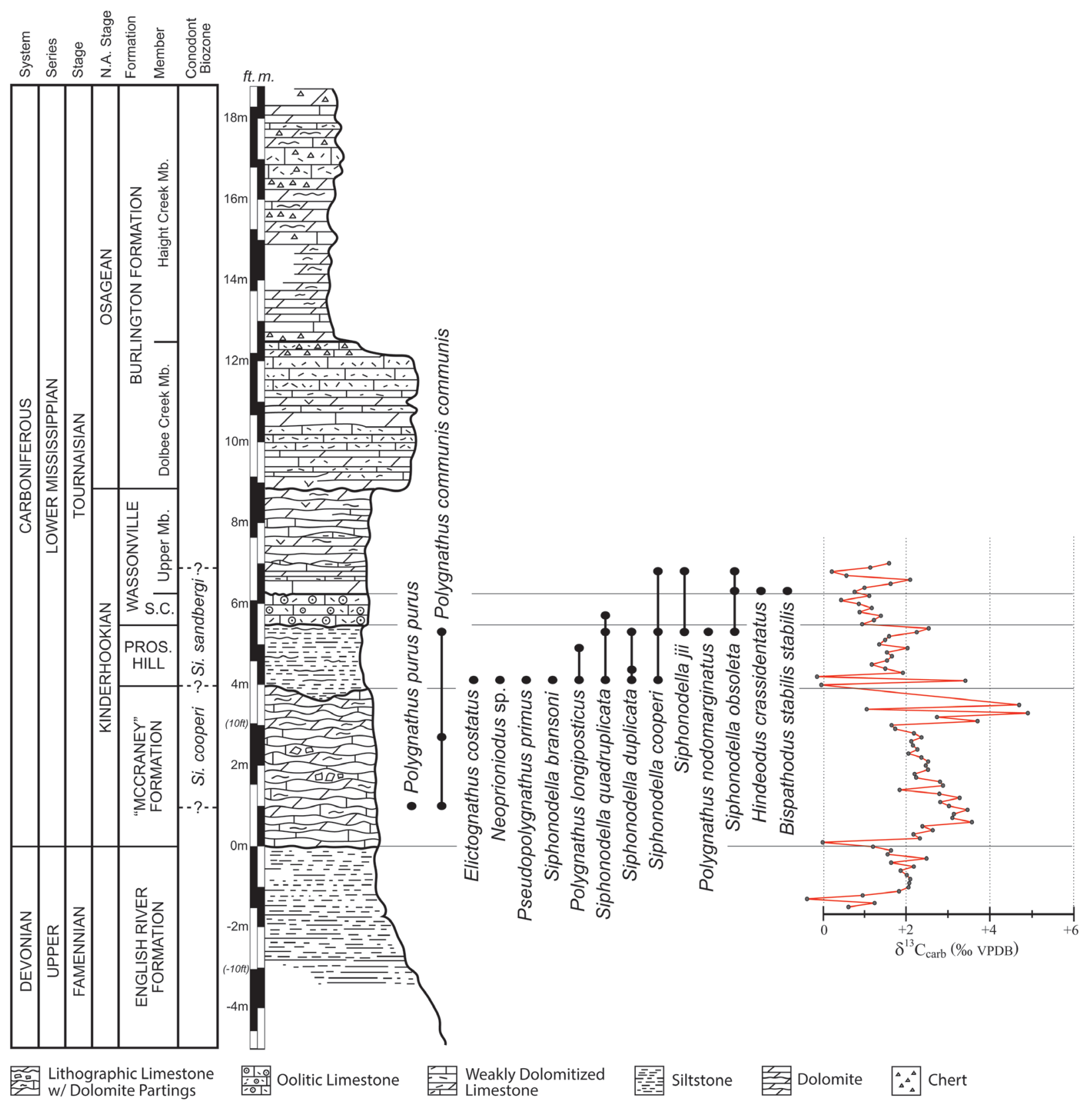

Figure 13. Conodont biostratigraphy and carbon isotope chemostratigraphy of the Starr's Cave Outcrop in Des Moines County, Iowa. Conodont occurrences are represented by small circles.

The Louisiana Limestone in its type area of eastern Missouri contains $\delta^{13} \mathrm{C}_{\text {carb }}$ values greater than $+6 \%$ (Cramer et al. 2008) indicative of the Hangenberg positive carbon isotope excursion (Kaiser et al. 2016). Therefore, the combined conodont and carbon isotope biochemostratigraphic data clearly demonstrate that the Louisiana Fm. in its type area records the Hangenberg Crisis, the Hangenberg positive carbon isotope excursion, and is at least in part within the P. kockeli conodont
Zone (Kaiser 2005, 2009; Cramer et al. 2008; Becker et al. 2016; Kaiser et al. 2016; Fig. 3 herein). In its type area the Louisiana Formation is typically devoid of siphonodellids, and is consistently overlain by the Hannibal Formation.

Samples from the McCraney Formation at its type locality in Kinderhook, Illinois has not produced conodont specimens. Most recently, Chauffe \& Guzman (1997) sampled the type section of the McCraney at McCraney 


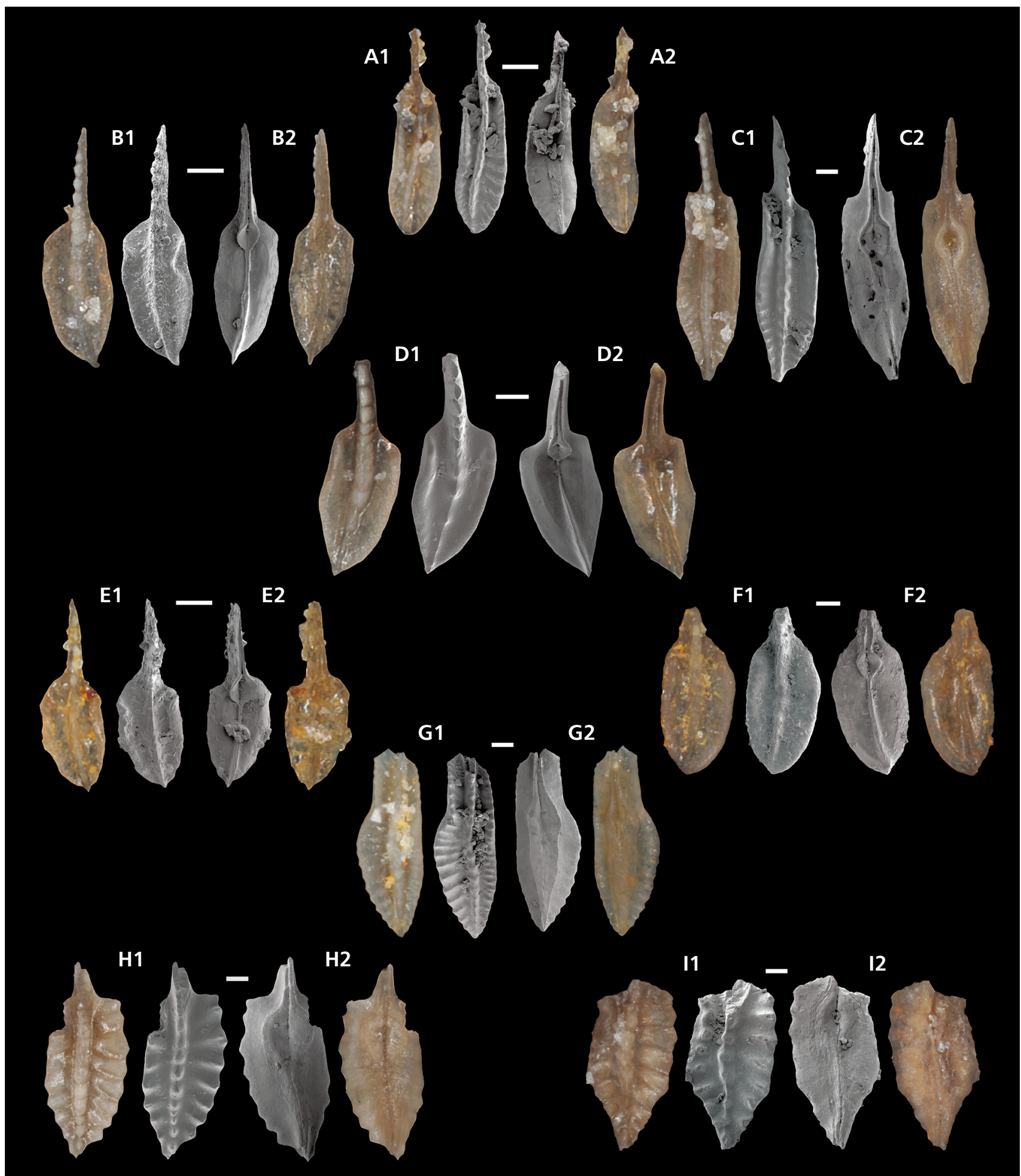

Figure 14. Conodonts from the Starr's Cave Outcrop. • A, C - Polygnathus longiposticus Branson \& Mehl, 1934a; A - [?] dextral $\mathrm{P}_{1}$ element, oral view (A1), aboral view (A2), "McCraney" Fm., 0.9-1.1 m, SUI 148269; C - dextral $\mathrm{P}_{1}$ element, oral view (C1), aboral view (C2), Prospect Hill Fm., 4.8-5.0 m, SUI 148270. • B, D, E - Polygnathus communis communis (Branson \& Mehl, 1934a); B - sinistral $\mathrm{P}_{1}$ element, oral view (B1), aboral view (B2), "McCraney" Fm., 2.6-2.8 m, SUI 148271; D - dextral $\mathrm{P}_{1}$ element, oral view (D1), aboral view (D2), Prospect Hill Fm., 5.2-5.4 m, SUI 148272; E - sinistral $\mathrm{P}_{1}$ element, oral view (E1), aboral view (E2), "McCraney Fm., 0.9-1.1 m, SUI 148273. • F - Polygnathus purus purus (Voges, 1959), dextral $P_{1}$ element, oral view (F1), aboral view (F2), "McCraney" Fm., 0.9-1.1 m, SUI 148274. • G - Siphonodella bransoni Ji, 1985; sinistral $\mathrm{P}_{1}$ element, oral view (G1), aboral view (G2), Prospect Hill Fm., 4.0-4.2 m, SUI 148275. • H - Polygnathus nodomarginatus E.R. Branson in Branson \& Meh1, 1934a; dextral $\mathrm{P}_{1}$ element) oral view (H1), aboral view (H2), "McCraney" Fm., 2.6-2.8 m, SUI 148276. • I - sinistral $\mathrm{P}_{1}$ element, oral view (D1), aboral view (D2), Prospect Hill Fm., 5.2-5.4 m, SUI 148277. 


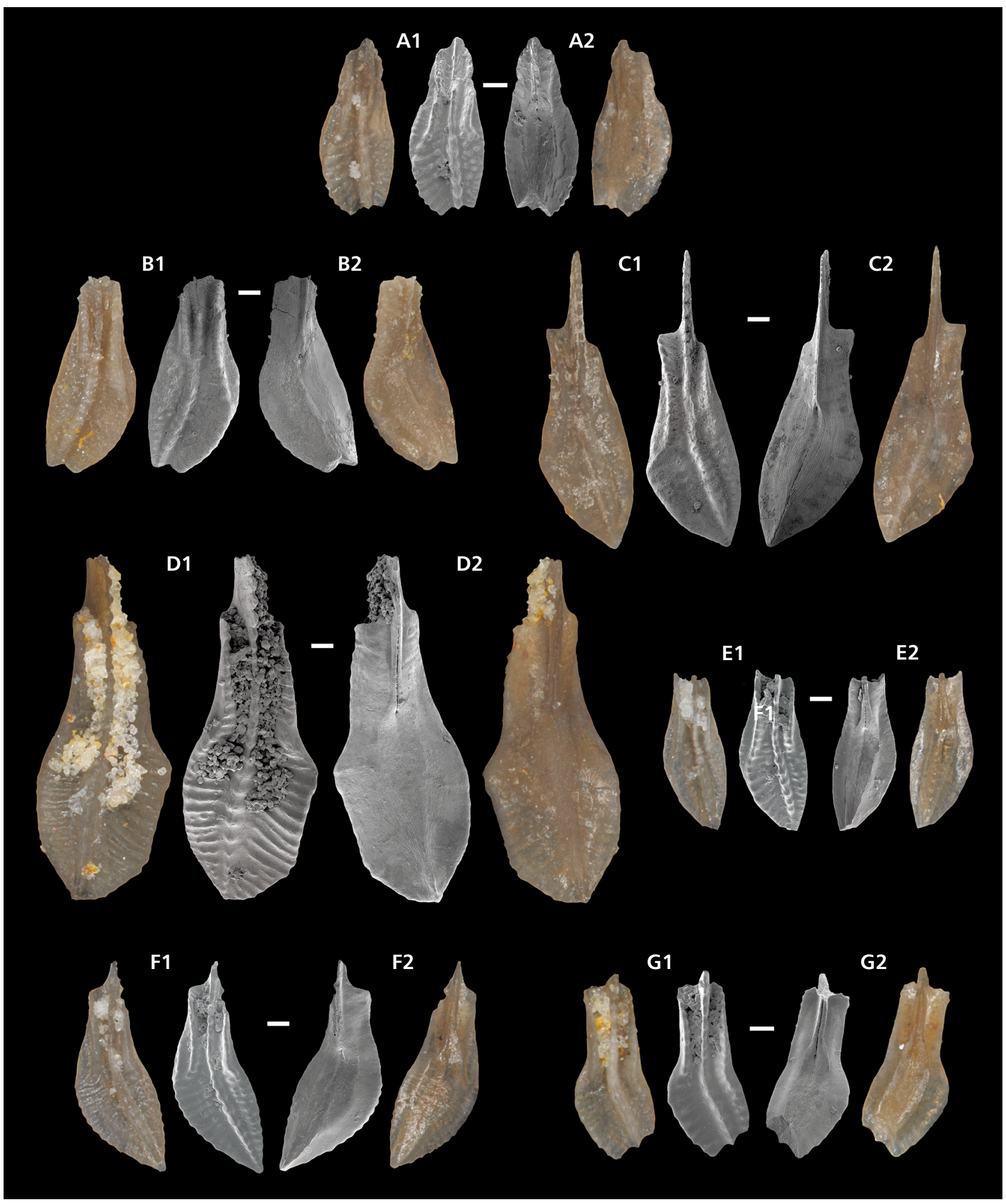

Figure 15. Conodonts from the Starr's Cave Outcrop. • A, E, G - Siphonodella cooperi Hass, 1959; A - sinistral $\mathrm{P}_{1}$ element, oral view (A1), aboral view (A2), Prospect Hill Fm., 5.2-5.4 m, SUI 148278; E - sinistral $P_{1}$ element, oral view (E1), aboral view (E2), Prospect Hill Fm., 5.2-5.4 m, SUI 148279; G - sinistral $\mathrm{P}_{1}$ element, oral view (G1), aboral view (G2), Prospect Hill Fm., 4.0-4.2 m, SUI 148280. • B, C - Siphonodella obsoleta Hass, 1959; B - dextral $\mathrm{P}_{1}$ element, oral view (B1), aboral view (B2), Upper Member, Wassonville Fm., 6.2-6.4 m, SUI 148281; C - sinistral $\mathrm{P}_{1}$ element, oral view (C1), aboral view (C2), Upper Member, Wassonville Fm., 6.7-6.8 m, SUI 148282. • D - Siphonodella duplicata (Branson \& Meh1, 1934a); dextral $\mathrm{P}_{1}$ element, oral view (D1), aboral view (D2), Prospect Hill Fm., 4.0-4.2 m, SUI 148283. • F - Siphonodella jii Becker et al., 2016; sinistral $P_{1}$ element, oral view (F1), aboral view (F2), Prospect Hill Fm., 5.2-5.4 m, SUI 148284. 


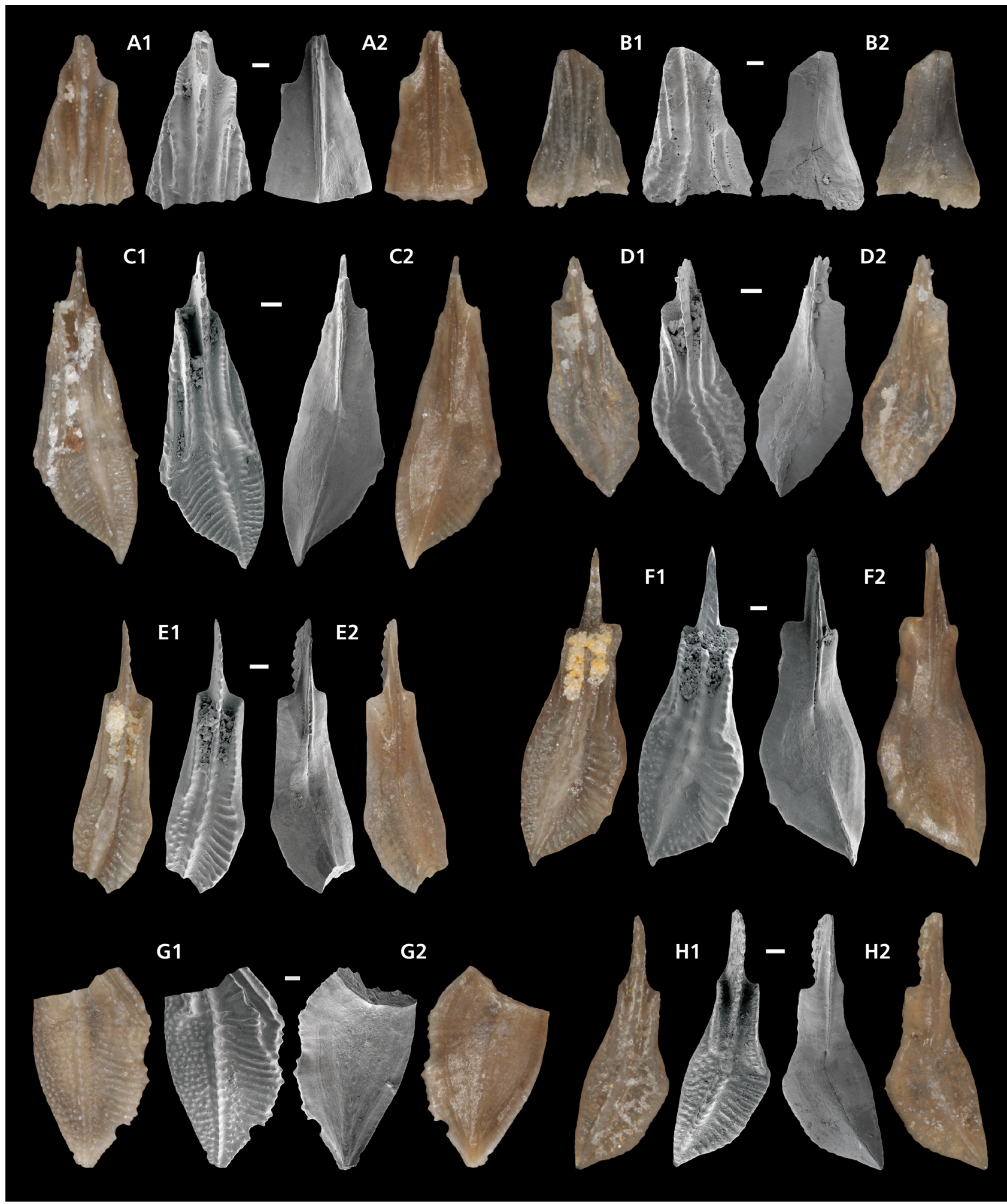

Figure 16. Conodonts from the Starr's Cave Outcrop. $-A-D$ - Siphonodella quadruplicata (Branson \& Mehl, 1934a); A - [?] sinistral $\mathrm{P}_{1}$ element, oral view (A1), aboral view (A2), Prospect Hill Fm., 5.2-5.4 m, SUI 148285; B - dextral $\mathrm{P}_{1}$ element, oral view (B1), aboral view (B2), Starr's Cave Member, Wassonville Fm., 5.6-5.8m, SUI 148286; C - sinistral $\mathrm{P}_{1}$ element, oral view (C1), aboral view (C2), Prospect Hill Fm., 5.2-5.4 m, SUI 148287; D - sinistral $\mathrm{P}_{1}$ element, oral view (D1), aboral view (D2), Prospect Hill Fm., 5.2-5.4 m, SUI 148288. • E-H - Siphonodella cooperi Hass, 1959; E - dextral $P_{1}$ element, oral view (E1), aboral view (E2), Prospect Hill Fm., 4.0-4.2 m, SUI 148289; F - dextral $P_{1}$ element, oral view (F1), aboral view (F2), Prospect Hill Fm., 4.0-4.2 m, SUI 148290; G - [?] dextral $P_{1}$ element, oral view (G1), aboral view (G2), Prospect Hill Fm., 5.2-5.4 m, SUI 148291; H - dextral $\mathrm{P}_{1}$ element, oral view (H1), aboral view (H2), Upper Member, Wassonville Fm., 6.7-6.9 m, SUI 148292. 


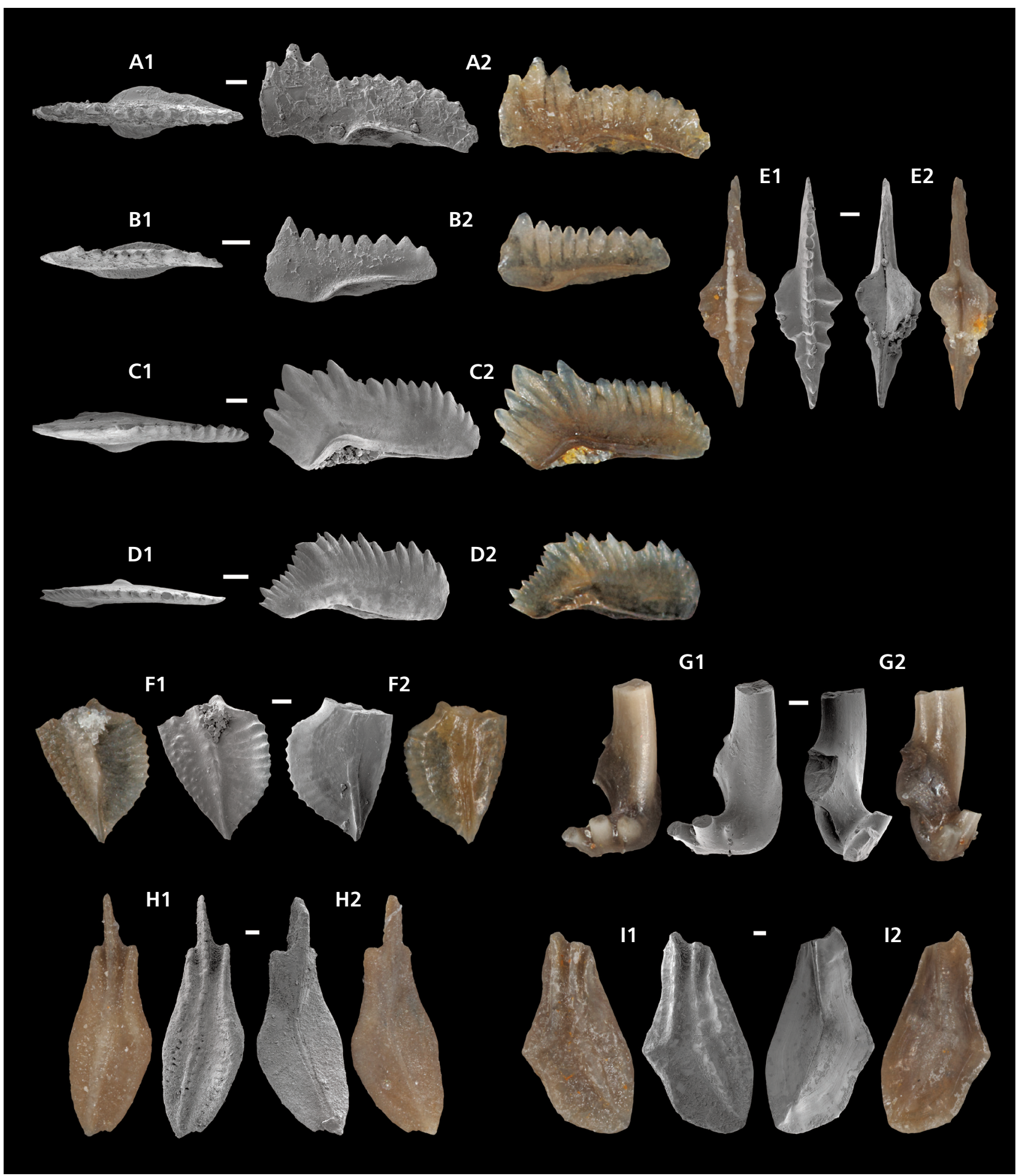

Figure 17. Conodonts from the Starr's Cave Outcrop. • A - Hindeodus crassidentatus (Branson \& Mehl, 1934a); dextral $\mathrm{P}_{1}$ element, oral view (A1), caudal view (A2), Upper Member, Wassonville Fm., 6.2-6.4 m, SUI 148293. • B - Bispathodus stabilis stabilis (Branson \& Mehl, 1934b); dextral $\mathrm{P}_{1}$ element, oral view (B1), caudal view (B2), Upper Member, Wassonville Fm., 6.2-6.4 m, SUI 148294. • C, D - Elictognathus costatus (Branson \& Mehl, 1934a); C - sinistral $P_{2}$ element, oral view (C1), caudal view (C2), Prospect Hill Fm., 4.0-4.2 m, SUI 148295; D - sinistral $P_{2}$ element, oral view (D1), caudal view (D2), Prospect Hill Fm., 4.0-4.2 m, SUI 148296. E - Pseudopolygnathus primus Branson \& Mehl, 1934a; ?sinistral $\mathrm{P}_{1}$ element, oral view (E1), aboral view (E2), Prospect Hill Fm., 4.0-4.2 m, SUI 148297. • F-I - Siphonodella sp.; F - dextral $\mathrm{P}_{1}$ element, oral view (F1), aboral view (F2), Prospect Hill Fm., 4.8-5.0 m, SUI 148298; H - dextral $\mathrm{P}_{1}$ element, oral view (H1), aboral view (H2), Upper Member, Wassonville Fm., 6.2-6.4 m, SUI 148299; I - sinistral $P_{1}$ element, oral view (I1), aboral view (I2), Upper Member, Wassonville Fm., 6.7-6.9 m, SUI 148300. • G Neoprioniodus sp., Prospect Hill Fm., 4.0-4.2 m, SUI 148301. 
North and recovered no conodonts from $25 \mathrm{~kg}$ of sample. No other conodont data have been published from the McCraney Formation at McCraney North (see discussion in Chauffe \& Guzman 1997). Conodonts recovered from the underlying Hannibal Formation at McCraney North include Elictognathus laceratus, Siphonodella duplicata, and $S$. cooperi, indicating a position no lower than the $S$. cooperi Zone for the base of the type McCraney. Across the river in Newark, Missouri (Fig. 1B), the McCraney Formation is in a stratigraphic position above the Choteau Formation (Fig. 4), is at the top of the Kinderhookian sequence, and contains the conodonts Siphonodella crenulata, Arismotaxis barbatus, Protognathodus prae- delicatus, and Gnathodus aff. G. punctatus (Chauffe \& Guzman 1997). Collectively, these demonstrate a significantly higher chronostratigraphic position than the Louisiana Formation in each of their type areas. Therefore, in this area of Missouri, the McCraney is latest Kinderhookian in age and does not correlate to any known portion of the Louisiana.

The H-28 core contains the entirety of the Hangenberg positive carbon isotope excursion including both the ascending and descending limbs (Fig. 5). The Hangenberg positive carbon isotope excursion (Kaiser 2005; Kaiser et al. 2006, 2008, 2016; Becker et al. 2016) begins in the uppermost Bispathodus ultimus Zone (Middle praesulcata

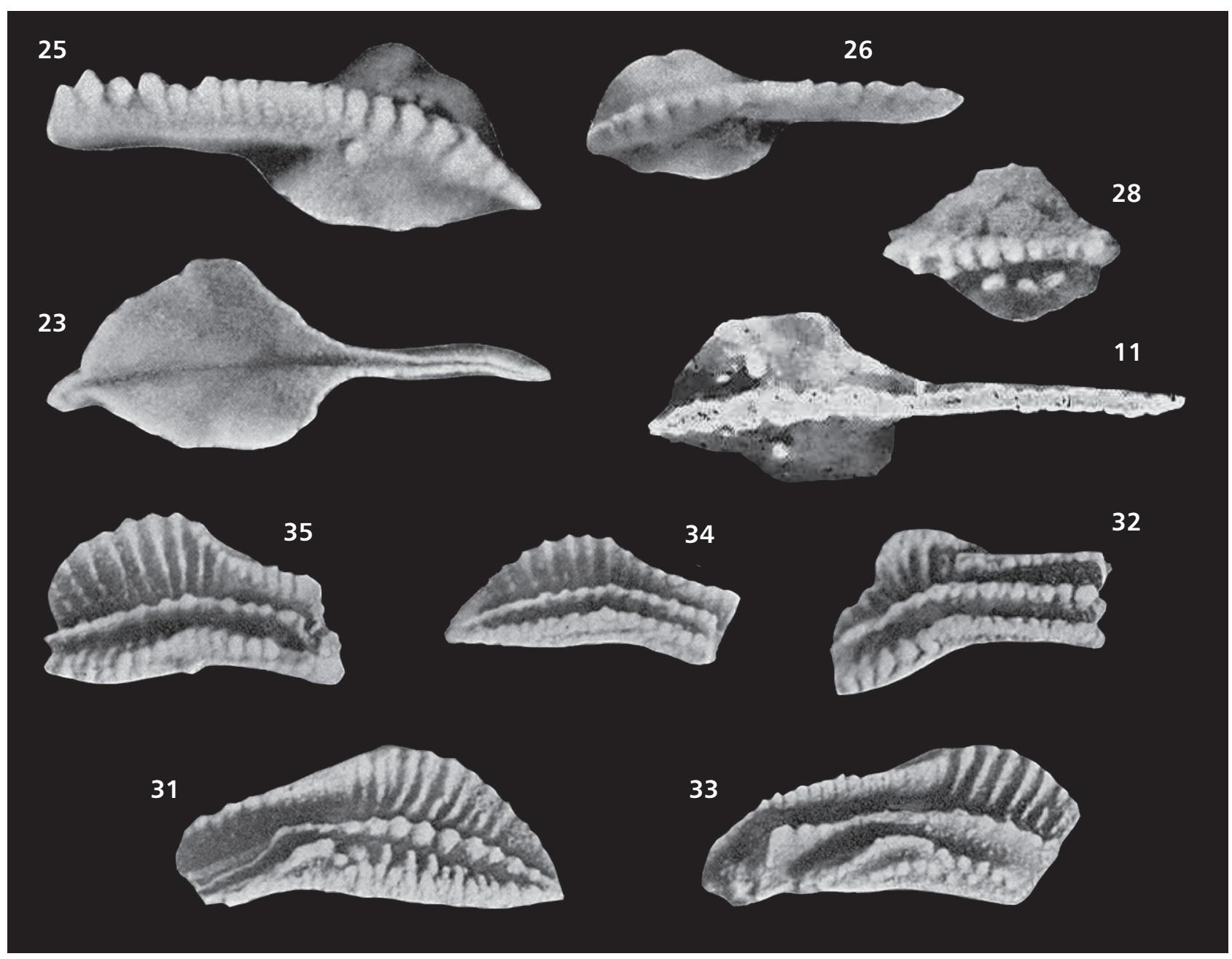

Figure 18. Re-illustrations of images from Scott \& Collinson (1961) and Straka (1968). Specimen numbers are the original designations from the original authors. Specimens 23, 25, 26, and 28 are from Scott \& Collinson (1961, pl. 1), images are 40x, and all specimens are from the Teneriffe School Section, Jersey County, Illinois. Specimens 31-35 are from Scott \& Collinson (1961, pl. 2), images are 40x, and all specimens are from Cascade Station, Burlington, Iowa. Specimen 11 is from Strata (1968, pl. 7), and image is 58x. $\bullet 11,23$, 25 - Protognathodus collinsoni Ziegler, 1969; 11 - sinistral $\mathrm{P}_{1}$ element,oral view, Wassonville Fm., SUI 125484; 23 - dextral $\mathrm{P}_{1}$ element, oral view, Louisiana Fm.; 25 - dextral $\mathrm{P}_{1}$ element, basal view, Louisiana Fm.. • 26 - Protognathodus meischneri Ziegler, 1969; sinistral $\mathrm{P}_{1}$ element, oral view, Louisiana Fm. 28 - Protognathodus kockeli (Bischoff, 1957), ?sinistral $P_{1}$ element, oral view, Louisiana Fm.. • 31, 32, 34, 35 - Siphonodella duplicata (Branson \& Mehl, 1934a), "McCraney" Fm.; 31 - [?] dextral $P_{1}$ element, oral view; 32 - sinistral $P_{1}$ element, oral view; 34 - sinistral $P_{1}$ element, oral view; 35 - sinistral $P_{1}$ element, oral view. 33 - Siphonodella cooperi Hass, 1959; dextral $\mathrm{P}_{1}$ element, oral view, "McCraney" Fm. 
Zone), and extends at least into the uppermost part of the P. kockeli Zone (sulcata Zone). The recognition of the onset of the Hangenberg excursion allowed us to tentatively place the $B$. ultimus and $P$. kockeli Zones in the core, and they fit well with the sequence of siphonodellids recovered from the Prospect Hill. Therefore, we can confidently say that the Hangenberg positive carbon isotope excursion is recorded by the upper English River and Louisiana formations in the $\mathrm{H}-28$ core.

The chronostratigraphic correlation of the end of the Hangenberg excursion remains enigmatic. The presence of $S$. cooperi Zone and S. sandbergi Zone conodonts in the Prospect Hill Fm. in H-28 core agrees well with prior biostratigraphy of the Prospect Hill in Iowa (Straka 1968), and requires that the interval from the P. kockeli Zone at least through the $S$. duplicata Zone be present in the Louisiana Fm. of the core. Similarly, the presence of both $S$. duplicata and S. cooperi in the "McCraney" within the city of Burlington (Cascade Station Section, Scott \& Collinson 1961, see below and Fig. 18) support elevated carbon isotope values at least into the $S$. duplicata Zone in the region.

The succession at Starr's Cave contains an identical stratigraphic succession as the H-28 core that includes the English River, "McCraney", Prospect Hill, Starr's Cave, Wassonville, and Burlington formations. The only difference in the stratigraphic succession between this locality and H-28 core is that the carbonate unit between the Prospect Hill and English River formations is named "McCraney" instead of Louisiana. This carbonate unit at Starr's Cave records $\delta^{13} \mathrm{C}$ values greater than $+5.0 \%$ (Fig. 13), which likely indicates a position somewhere within the Hangenberg carbon isotope excursion interval. Scott \& Collinson (1961) recovered conodonts from the "McCraney" at Cascade Station $[<10$ miles $(16 \mathrm{~km})$ from Starr's Cave] within the city of Burlington, and they are re-illustrated here in Fig. 18 using their original specimen numbers from the original plates. Whereas they originally identified all of these specimens as $S$. cooperi, with the exception of specimen 32 as $S$. duplicata, we suggest that only specimen 33 has a demonstrably nodose interior platform and suggest that all specimens should be designated $S$. duplicata, with the exception being specimen 33 as S. cooperi. All five of these specimens were recovered from an interval "three to six feet above the base of the Louisiana" (Scott \& Collinson 1961, p. 119). Conodonts recovered from the Prospect Hill at Starr's Cave demonstrate a position within the $S$. sandbergi Zone which matches perfectly with the identification of S. duplicata and S. cooperi in the "McCraney" by Scott \& Collinson (1961). This position of the "McCraney" in Burlington Iowa ( $S$. duplicata and S. cooperi), is considerably below the McCraney at Newark, Missouri (Chauffe \& Guzman 1997), and matches well the upper portion of the Louisiana in the H-28 core (compare Figs 5 and 13). Therefore, the unit referred to as "McCraney" at Starr's Cave is more likely correctly identified as the upper Louisiana Fm., even if it does not represent the entire Louisiana Fm. preserved in the H-28 core.

\section{Conclusions}

The H-28 core and Starr's Cave outcrop were examined using integrated conodont biostratigraphy and carbon isotope chemostratigraphy. These analyses resulted in the detection of the Hangenberg positive carbon isotope excursion and returned a diverse fauna of siphonodellids. Combined with data from the type areas in Missouri and Illinois we can conclude that the carbonate unit beneath the Prospect Hill Formation in the H-28 core is undoubtedly the Louisiana Formation, not the "McCraney". Similarly, the carbonate unit below the prospect Hill Formation referred to as the "McCraney" at Starr's Cave is likely the upper part of the Louisiana. This calls into question whether or not the name McCraney should be applied to any stratigraphic units in the State of Iowa.

The combined data presented herein demonstrate that the Devonian-Carboniferous boundary interval is contained in an extremely expanded section in the type Mississippian tri-state area of Iowa, Missouri, and Illinois. Whereas most sections in the world where the Hangenberg positive carbon isotope excursion has been recovered contain a few meters of elevated positive $\delta^{13} \mathrm{C}_{\text {carb }}$ values, the tri-state area of the upper Mississippi Valley has nearly 20 meters of this critical interval in the co-evolution of ocean-atmosphere-biosphere Earth system. Furthermore, the comparatively carbonate-rich succession of the tristate area allows for more complete and detailed carbon isotope chemostratigraphy and conodont biostratigraphy than many other regions in the world. Collectively, this study demonstrates the importance of this part of the U.S. Mid-continent to any potential re-evaluation of the base Carboniferous GSSP and choice of combined biological and chemical markers for global chronostratigraphic correlation.

\section{Acknowledgments}

This work was partially supported by the USGS National Cooperative Geological Mapping Program under STATEMAP award numbers G16AC00196 (2016), G17AC00258 (2017), \& G18AC00194 (2018), and by National Science Foundation grants GP-IMPACT-1600429 and CAREER-1455030. This research was completed as an undergraduate senior thesis project in Geoscience at the University of Iowa, Department of Earth and Environmental Sciences. 


\section{References}

Becker, R.T., Kaiser, S.I. \& Aretz, M. 2016. Review of chrono-, litho, and biostratigraphy across the global Hangenberg Crisis and Devonian Carboniferous Boundary, 355-386. In Becker, R.T., Königshof, P. \& Brett, C.E. (eds) Devonian Climate, Sea Level and Evolutionary Event. Geological Society London, Special Publication 423.

DOI 10.1144/SP423.10

Bischoff, G. 1957. Die Conodonten-Stratigraphie des rhenoherzynischen Unterkarbons mit Berücksichtigung der Wocklumeria-Stufe und der Devon/Karbon-Grenze. Abhandlungen des Hessichen Landesamtes für Bodenforschung 19, 1-64.

Branson, E.R. 1934. Conodonts from the Hannibal formation of Missouri. University of Missouri Studies 8(4), 301-334.

Branson, E.B. \& Mehl, M.G. 1934a. Conodonts from the Bushberg sandstone and equivalent formations of Missouri. University of Missouri Studies 8(4), 265-299.

Branson, E.B. \& Mehl, M.G. 1934b. Conodonts from the Grassy Creek shale of Missouri. University of Missouri Studies 8(3), 171-259.

Chauffe, K.M. \& Guzman, M. 1997. Conodonts from the McCraney limestone and the McCraney-Chouteau limestone transition beds (Kinderhookian, Lower Carboniferous) in northeastern Missouri and west-central Illinois, U.S.A. Micropaleontology 43, 221-252. DOI 10.2307/1485826

Chauffe, K.M. \& Nichols, P.A. 1995. Multielement conodonts species from the Louisiana Limestone (Upper Devonian) of west-central Illinois and north-eastern Missouri, U.S.A. Micropaleontology 41, 171-186. DOI 10.2307/1485950

Cooper, C.L. 1939. Conodonts from a Bushberg-Hannibal horizon in Oklahoma. Journal of Paleontology 13, 379-422.

Corradini, C., Kaiser, S.I., Perri, M.C. \& Spalletta, C. 2011. Protognathuodus (Conodonta) and its potential as a tool for defining the Devonian/Carboniferous Boundary. Rivista Italiana di Paleontologia e Stratigrafia 117, 15-28.

Corradini, C., Spalletta, C., Mossoni, A., Matyja, H. \& Over, D.J. 2017. Conodont across the Devonian/Carboniferous boundary: a review and implication for the redefinition of the boundary and a proposal for an updated conodont zonation. Geological Magazine 154, 888-902. DOI 10.1017/S001675681600039X

Cramer, B.D., Saltzman, M.R., Day, J.E. \& Witzke, B.J. 2008. Record of the Late Devonian Hangenberg Global Positive Carbon Isotope Excursion in an Epeiric Sea Setting: Carbonate Production, Organic Carbon Burial and Paleoceanography During the Late Famennian, 103-118. In Holmden, H. \& Pratt, B.R. (eds) Dynamics of Epeiric Seas: Sedimentological, paleontological and Geochemical Perspectives. Geological Association of Canada, Special Paper 48.

Davydov, W.I., Korn, D. \& Schmitz, M.D. 2012. The Carboniferous period, 603-651. In Gradstein, F.M., OGG, J.G., Schmitz, M. \& OGG, G. (eds) The Geologic Time Scale 2012. Elsevier, Amsterdam.

DOI 10.1016/B978-0-444-59425-9.00023-8

HARRIS, E.H. JR. 1947. Subsurface stratigraphy of the Kinder- hook and Osage Series in southeastern Iowa. 164 pp. Ph. D. thesis, University of Iowa, Iowa city, U.S.A.

DOI 10.17077/etd.005128

Hass, W.H. 1959. Conodonts from the Chappel Limestone of Texas. United States Geological Survey Professional Paper 249-J, 365-399. DOI 10.3133/pp294J

Heckel, P.H. 2001. Stratigraphy and biostratigraphy of the Mississippian Subsystem (Carboniferous System) in its type region, the Mississippi River Valley of Illinois, Missouri, and Iowa. International Union of Geological Sciences Subcommission on Carboniferous Stratigraphy Guidebook for Field Conference, September 8-13, 2001. 120 pp. Illinois State Geological Survey.

Hogancamp, N.J., Stolfus, B.M., Cramer, B.D. \& Day, J.E. 2019. A revised conodont zonation of the Tournaisian (Kinderhookian to Lower Osagean) and implications for stratigraphic correlations in North America, 11-17. In CRAMER, B.D., Clark, R.J. \& DaY, J.E. (eds) The Devonian-Carboniferous Boundary in the Type Area of the Mississippian, Iowa Geological Survey, Guidebook 30.

Hudde, J.W. 1934. Conodonts from the New Albany Shale of Indiana. Bulletin American Paleontology 21(72), 1-136.

Jeppsson, L. \& Anehus, R. 1995. A Buffered Formic Acid Technique for Conodont Extraction. Journal of Paleontology 69(4), 790-794. DOI 10.1017/S0022336000035319

JI, Q. 1985. Study on the phylogeny, taxonomy, zonation and biofacies of Siphonodella (Conodonta). Bulletin of the Institute of Geology 11, 51-75.

KAISER, S.I. 2005. Mass extinctions, climatic and-oceanographic changes at the Devonian-Carboniferous boundary. 156 pp. Ph.D. thesis, Fakultät für Geowissenschaften, RuhrUniversität Bochum, Germany.

Kaiser, S.I. 2009. The Devonian/Carboniferous stratotype section La Serre (Montagne Noire) revisited. Newsletters on Stratigraphy 43, 195-205.

DOI 10.1127/0078-0421/2009/0043-0195

KAiser, S.I. \& Becker, R.T. 2007. The required revision of the Devonian-Carboniferous boundary, p. 95. In WANG, Y., Zhang, H. \& Wang, X. (eds) XVI International Congress on the Carboniferous and Permian, Abstracts. Journal of Stratigraphy 31.

Kaiser, S.I. \& Corradini, C. 2008. Should the Devonian/ Carboniferous boundary be redefined? SDS Newsletter 23, $55-56$.

Kaiser, S.I., Aretz, M. \& Becker, R.T. 2016. The global Hangenberg Crisis (Devonian-Carboniferous transition): review of a first-order mass extinction, 387-437. In BECKER, R.T., Königshof, P. \& Brett, C.E. (eds) Devonian Climate, Sea Level and Evolutionary Event. Geological Society of London, Special Publication 423. DOI 10.1144/SP423.9

Kaiser, S.I., Steuber, T. \& Becker, R.T. 2008. Environmental change during the Late Famennian and Early Tournaisian (Late Devonian-Early Carboniferous) - implications from stable isotopes and conodont biofacies in southern Europe, 241-260. In Aretz, M., Herbig, H.-G. \& Somerville, I.D. (eds) Carboniferous Platforms and Basins. Geological Journal 43. DOI 10.1002/gj.1111 
Kaiser, S.I., Becker, R.T., Spalletta, C. \& Steuber, T. 2009. High-resolution conodont stratigraphy, biofacies, and extinctions around the Hangenberg Event in pelagic successions from Austria, Italy, and France. Palaeontographica Americana 63, 97-139.

Kaiser, S.I., Becker, R.T., Steuber, T. \& Aboussalam, Z.S. 2011. Climate-controlled mass extinctions, facies, and sealevel changes around the Devonian-Carboniferous boundary in the eastern Anti-Atlas (SE Morocco). Paleogeography, Paleoclimatology, Paleoecology 310, 340-364.

DOI 10.1016/j.palaeo.2011.07.026

Kaiser, S.I., Steuber, T., Becker, R.T. \& JoAchimski, M.M. 2006. Geochemical evidence for major environmental change at the Devonian-Carboniferous boundary in the Carnic Alps and the Rhenish Massif. Palaeogeography, Palaeoclimatology, Palaeoecology 240, 146-160.

DOI 10.1016/j.palaeo.2006.03.048

Keyes, C.R. 1895. Geology of Lee County, Iowa Geological Survey, Annual Report 3, 305-409.

DOI 10.17077/2160-5270.1314

Klapper, G. \& Phillip, G. M. 1971. Devonian conodont apparatuses and their vicarious skeletal elements. Lethaia 4, 429-452. DOI 10.1111/j.1502-3931.1971.tb01865.x

Lane, H.R. 1978. The Burlington Shelf (Mississippian, northcentral United States). Geologica et Palaeontologica 12, 165-176.

Lane, H.R. \& Brenckle, P.L. 2001. Type Mississippian subdivisions and biostratigraphic succession, 83-107. In Heckel, P.H. (ed.) Stratigraphy and biostratigraphy of the Mississippian Subsystem (Carboniferous System) in its type region, the Mississippi River Valley of Illinois, Missouri, and Iowa. International Union of Geological Sciences, Subcommission on Carboniferous Stratigraphy, Guidebook for Field Conference.

LAudon, L.R. 1931. The stratigraphy of the Kinderhook Series of Iowa. Iowa Geological Survey, Annual Report 35, 333-451. DOI 10.17077/2160-5270.1250

Moore, R.C. 1928. Early Mississippian formations in Missouri. Missouri Bureau of Geology and Mines, 2 ${ }^{\text {nd }}$ Series 21, p. 283.

Paproth, E., Feist, R. \& Flajs, G. 1991. Decision on the Devonian-Carboniferous boundary stratotype. Episodes 14, 331-336. DOI 10.18814/epiiugs/1991/v14i4/004

Rhodes, F.H.T., Austin, R.L. \& Druce, E.C. 1969. British Avonian (Carboniferous) conodont faunas and their value in local and intercontinental correlation. Bulletin British Museum of Natural History (Geology) Supplement 5, 1-313.

Sandberg, C.A., Streel, M. \& Scott, R.A. 1972. Comparison between conodont zonation and spore assemblages at the Devonian-Carboniferous boundary in the western and central United States and in Europe. Compte Rendu $7^{\text {th }}$ International Congress of Carboniferous Stratigraphy and Geology, 23-28 August, Krefeld, Germany, 179-203.

Sandberg, C.A., Zeigler, W., Leuteritz, K. \& Brill, S.M. 1978. Phylogeny, speciation, and zonation of Siphonodella (Conodonta, Upper Devonian and Lower Carboniferous). Newsletters on Stratigraphy 7(2), 102-120.

DOI $10.1127 / \operatorname{nos} / 7 / 1978 / 102$
Saltzman, M.R. 2002, Carbon isotope ( $\delta 13 C)$ stratigraphy across the Silurian-Devonian transition in North America: Evidence for a perturbation of the global carbon cycle. Palaeogeography, Palaeoclimatology, Palaeoecology 187, 83-100. DOI 10.1016/S0031-0182(02)00510-2.

Saltzman, M.R. \& Thomas, E. 2012. Carbon isotope stratigraphy, 207-232. In Gradstein, F.M., OGG, J.G., Schimtz, M. \& OGG, G. (eds) The Geologic Time Scale 2012. Elsevier, Amsterdam. DOI 10.1016/S0031-0182(02)00510-2

Scott, A.J. \& Collinson, C. 1961. Conodont faunas from the Louisiana and McCraney Formations of Illinois, Iowa, and Missouri. Twenty-sixth Annual Field Conference, Kansas Geological Survey, 110-141.

Sepkoski, J.J. JR. 1996. Patterns of Phanerozoic extinction: a perspective from global data bases, 35-51. In WALLISER, O.H. (ed.) Global Events and Event Stratigraphy in the Phanerozoic. Springer, Berlin.

DOI 10.1007/978-3-642-79634-0 4

Spalletta, C., Perri, M.C., Over, J.D. \& Corradini, C. 2017. Famennian (Upper Devonian) conodont zonation: revised global standard. Bulletin of Geosciences 92, 31-57. DOI 10.3140/bull.geosci.1623

Stainbrook, M.A. 1950. The fauna and correlation of the McCraney limestone of Iowa and Illinois. American Journal of Science 248, 194-213. DOI 10.2475/ajs.248.3.194

StrakA, J.J. 1968. Conodont zonation of the Kinderhookian Series, Washington County, Iowa. University of Iowa Studies in Natural History 21(2), 1-71.

Thomas, L.A. 1949. Devonian-Mississippian Formations of southeast Iowa. Geological Society of America Bulletin 60, 403-138. DOI 10.1130/0016-7606(1949)60[403:DFOSI]2.0.CO;2

Thompson, T.L. \& Fellows, L.D. 1970. Stratigraphy and conodont biostratigraphy of Kinderhookian and Osagean (Lower Mississippian) rocks of southwester Missouri \& adjacent areas. Missouri Geological Survey and Water Resources, Report of Investigations 45, 1-263.

Voges, A. 1959. Conodonten aus dem Unterkarbon I und II (Gattendorfia- und Pericyclus-Stufe) des Sauerlandes. Paläontologische Zeitschrift 33, 266-314. DOI 10.1007/BF02987939

Wachter, E.A. \& Hayes, J.M. 1985. Exchange of oxygen isotopes in carbon dioxide-phosphoric acid systems. Chemical Geology Isotope Geoscience Section 52, 365-374. DOI 10.1016/0168-9622(85)90046-6

Walliser, O.H. 1984. Pleading for a natural D/C-boundary. Courier Forschungsinstitut Senckenberg 67, 241-246.

Walliser, O.H. 1996. Global events in the Devonian and Carboniferous, 255-250. In Walliser, O.H. (ed.) Global Events and Event Stratigraphy in the Phanerozoic. Springer, Berlin. DOI 10.1007/978-3-642-79634-0_11

Weller, S. 1900. The succession of fossil faunas in the Kinderhook beds at Burlington, Iowa. Iowa Geological Survey, Annual Report 10, 63-79.

Weller, S. 1906. Kinderhook faunal studies, IV, The faunas of the Glen Park limestone, St. Louis Academy of Science 16, 435-471.

Weller, J.M. \& Sutton, A.H. 1940. Mississippian border of the 
Eastern Interior Basin, American Association of Petroleum Geologists Bulletin 24, 765-858.

DOI 10.1306/3D933206-16B1-11D7-8645000102C1865D

WiLLIAms, J. S. 1943. Stratigraphy and fauna of the Louisiana limestone of Missouri, United States Geological Survey Professional Paper 203, 1-129. DOI 10.3133/pp203

WitzKe, B.J. 2002. Regional stratigraphic relations of the Burlington-Keokuk formations (Mississippian) across the central U.S. and the nature of the sub-Burlington disconformity. Geological Society of America, Abstracts with Programs 34(2), A-40.

WitzKe, B.J. \& BunKer, B.J. 1996. Relative sea-level changes during Middle Ordovician through Mississippian deposition in the Iowa area, North American craton, 307-330. In Witzke, B.J., Ludvigson, G.A. \& DAy, J. (eds) Paleozoic Sequence Stratigraphy: Views from the North American Craton. Geological Society of America Special Paper 306. DOI 10.1130/0-8137-2306-X.307

Witzke, B.J. \& Bunker, B.J. 2001. Bedrock stratigraphy in the Burlington area. Geological Society of Iowa Guidebook 71, 9-19.

Witzke, B.J. \& Bunker, B.J. 2002. Bedrock geology in the Burlington area, southeast Iowa, 23-51. In WitzKe, B.J.,
Tassier-Surine, S.A., Anderson, R.R., Bunker, B.J. \& Artz, J.A. (eds) Pleistocene, Mississippian, \& Devonian Stratigraphy of the Burlington, Iowa, Area. Iowa Geological Survey Guidebook 23.

Witzke, B.J., McKay, R.M., Bunker, B.J. \& Woodson, F.J. 1990. Stratigraphy and paleoenvironments of Mississippian strata in Keokuk and Washington counties, southeast Iowa. Iowa Department of Natural Resources, Geological Survey Bureau, Guidebook Series 10, p. 105.

Witzke, B.J., Tassier-Surine, S.A., Anderson, R.R., Bunker, B.J. \& Artz, J.A. 2002. Pleistocene, Mississippian, \& Devonian Stratigraphy of the Burlington, Iowa, Area. Iowa Geological Survey Guidebook 23, 23-51.

Workman, L.E. \& Gillette, T. 1956. Subsurface stratigraphy of the Kinderhook Series in Illinois. Illinois State Geological Survey, Report of Investigations 189, p. 46.

Zhuravlev, A.V. \& Plotitsyn, A.N. 2017. The symmetry of the rostrum as a key to taxonomy of advanced Siphonodella (Conodonta, Early Carboniferous). Stratigraphy 14, 457-474. DOI 10.29041/strat.14.1-4.457-474

ZIEGLER, W. 1969. Eine neue Conodontenfauna aus dem höchsten Oberdevon. Fortschritt Geologie Rheinland und Westfalen 17, 343-360. 